\title{
Wireless Performance of a Fully Passive Neurorecording Microsystem Embedded in Dispersive Human Head Phantom
}

\author{
Helen N. Schwerdt, Junseok Chae \\ Electrical, Computer, and Energy Engineering \\ Arizona State University \\ Tempe, AZ, USA
}

\begin{abstract}
This paper reports the wireless performance of a biocompatible fully passive microsystem implanted in phantom media simulating the dispersive dielectric properties of the human head, for potential application in recording cortical neuropotentials. Fully passive wireless operation is achieved by means of backscattering electromagnetic (EM) waves carrying $3^{\text {rd }}$ order harmonic mixing products $\left(2 f_{0} \pm f_{m}=4.4-4.9 \mathrm{GHz}\right)$ containing targeted neuropotential signals $\left(f_{m} \approx 1-1000 \mathrm{~Hz}\right)$. The microsystem is enclosed in $4 \mu \mathrm{m}$ thick parylene-C for biocompatibility and has a footprint of $4 \mathrm{~mm} \times 12 \mathrm{~mm} \times 500 \mu \mathrm{m}$. Preliminary testing of the microsystem implanted in the lossy biological simulating media results in signal-to-noise ratio's (SNR) near $22(\mathrm{SNR} \approx 38$ in free space) for millivolt level neuropotentials, demonstrating the potential for fully passive wireless microsystems in implantable medical applications.
\end{abstract}

\section{INTRODUCTION}

In order to advance wireless biomedical implant technology, the safety and durability of the internal electronics is of utmost importance. For cortical brain recording applications, potential hazards introduced by implanted circuitry severely limit their clinical manifestation. Fully passive circuitry may alleviate many of the risks related to heat dissipation and potential failure of internal power sources, regulators, and/or harvesters. The fully passive device, presented herein, excludes any integrated power sources and transmits targeted neuropotential signals wirelessly by means of microwave backscattering (Fig. 1).

Previous testing of the fully passive wireless microsystem demonstrated a sensitivity of $\sim 500 \mu \mathrm{V}_{\mathrm{pp}}\left(V_{m}\right)$ as recorded from a frog's sciatic nerve and bandwidth $\left(f_{m}\right)$ of $5-2000 \mathrm{~Hz}$ [1], [2]. However, prior testing did not take into account the inhomogeneous tissue enclosing the microsystem in its intended application that would significantly alter penetrating EM signals.

\section{MATERIALS AND METHODS}

Miniaturization of the on-chip implant antenna is achieved by use of an electrically small slot antenna operating at higher microwave frequencies. Additional onboard circuitry includes 3 MIM (Metal-Insulator-Metal) capacitors (1 bypass and 2 loading capacitors) and 2 off-chip varactors (Fig. 2(a)).
Félix A. Miranda

Antenna and Optical Systems Branch

NASA Glenn Research Center

Cleveland, $\mathrm{OH}, \mathrm{USA}$

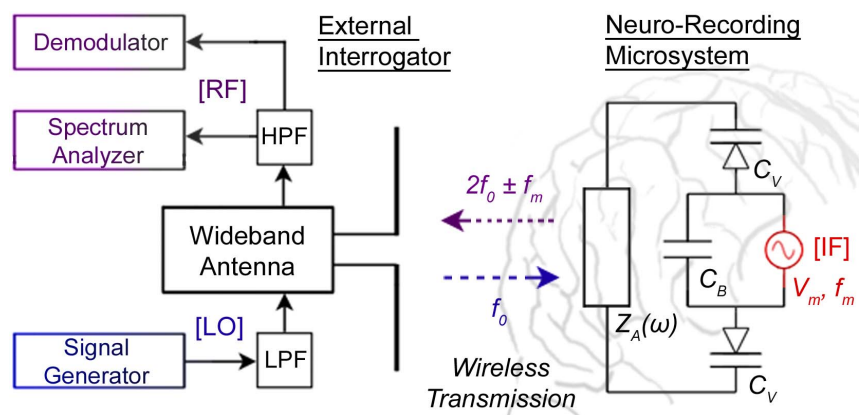

Figure 1. Simplified schematic of fully passive wireless operation. At the external interrogator (left), a signal generator supplies the $f_{0}$ local oscillator (LO) carrier that is low pass filtered (LPF) and wirelessly transmitted via wideband antenna to the microsystem (right) antenna $\left(Z_{A}(\omega)\right)$, which then backscatters $3^{\text {rd }}$ order harmonic RF signals $\left(2 f_{0} \pm f_{m}\right)$. The RF signals are received by the external antenna and fed into a high pass filter (HPF) and demodulated to baseband $\left(f_{m}\right)$.

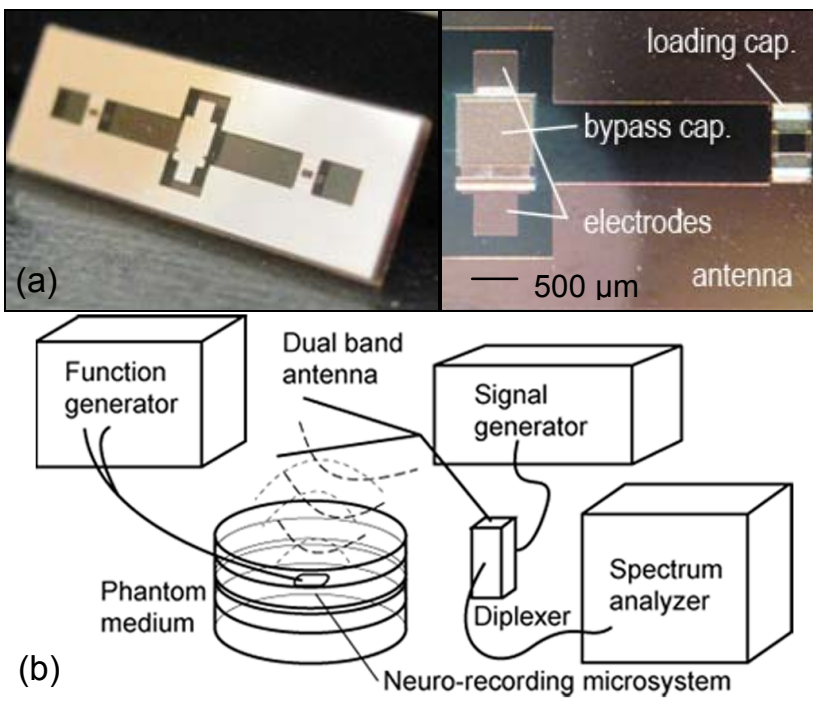

Figure 2. (a) Neurorecording microsystem (close up on right), measures $4 \times 12 \times 0.5 \mathrm{~mm}^{3}$ and integrates on-chip antenna, MIM capacitors, and electrodes connecting $V_{m}$ input to varactors. (b) Setup for wireless testing as embedded in phantom medium.

Capacitive loading by fabricated MIM capacitors permits dual band operation at the incident frequency $\left(f_{0}\right)$ and backscatter frequency $\left(2 f_{0} \pm f_{m}\right)$. An external interrogator supplies the 
fundamental carrier $\left(P_{0} @ f_{0}\right)$ signal to activate the microsystem's mixing and backscattering functions. Varactors retrieve this induced carrier $\left(P_{0} @ f_{0}\right)$ along with the internal neuropotential $\left(V_{m} @ f_{m}\right)$ signals to generate 3rd order harmonic mixing products $\left(2 f_{0} \pm f_{m}\right)$ that are then backscattered by the onchip antenna to the external interrogator, where the neuropotential signal is recovered (Fig. 2(b)).

The phantom medium is composed of multiple strata mimicking the complex permittivity characteristics of skin, bone, dura, gray matter, and white matter layers of the average human head (Fig. 3) [3]. Open-ended coaxial probe measurements $(85070 \mathrm{D}$, Agilent) of the various phantom layers are performed to ensure their permittivity values closely correspond to the reported values for real human tissues (Fig. 4) [4].

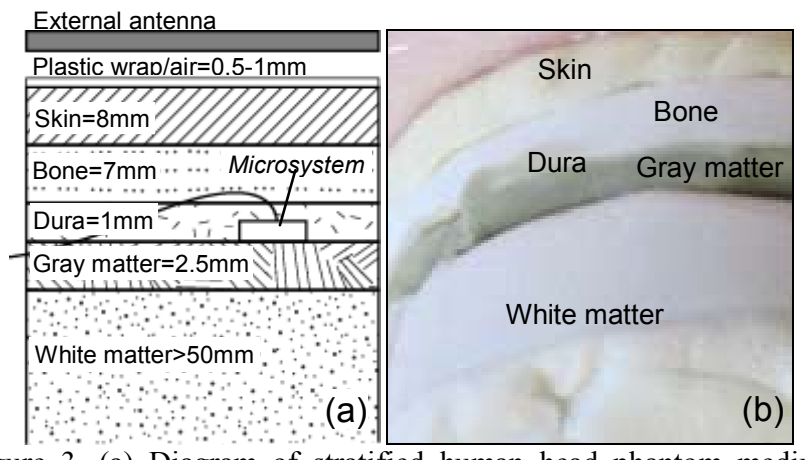

Figure 3. (a) Diagram of stratified human head phantom medium (with individual thicknesses) and (b) cross section of the phantom medium assembly.

During wireless testing, the neurorecording microsystem is embedded into the dura stratum of the phantom and the complete phantom is enclosed by plastic wrap to maintain an insulating barrier from the external interrogator (the external antenna is placed in direct contact with the plastic wrap for a total wireless separation of near $1 \mathrm{~mm}$ ). Emulated neuropotential signals $\left(V_{m}=0-50 \mathrm{mV}_{\mathrm{pp}}, f_{m}=5-1000 \mathrm{~Hz}\right)$, are applied to a twisted pair of insulated feed-through wires connected to the front of the microsystem. Local oscillator (LO) power $\left(P_{0}=0-20 \mathrm{dBm}\right)$ is supplied from a signal generator (8341A, HP) to supply the carrier. The SNR of the backscattered 3rd order harmonics $\left(2 f_{0} \pm f_{m}\right)$ from the microsystem is quantified by the ratio between the amplitude of the $\pm f_{m}$ sidebands around $2 f_{0}$ as visualized in the spectrum analyzer (E4448A, Agilent) with an average noise floor of $136 \mathrm{dBm}$ (Fig. 5(a)). The average observed SNR for the three different types of microsystems (low resistivity silicon, high resistivity silicon, and glass substrates) tested in air and in the phantom medium are summarized in Fig. 5(b). The microsystem based on high resistivity silicon and glass substrates produced a SNR of greater than $20 \mathrm{~dB}$ inside the phantom, whereas devices based on low resistivity silicon fail to generate any wireless response. Future work will involve enhancing the sensitivity of the microsystem and minimizing noise in the external demodulator, as will be delineated in the full paper.
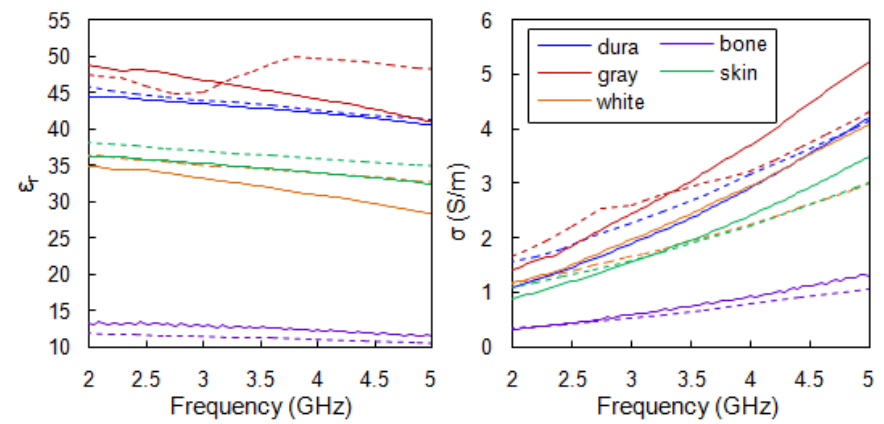

Figure 4. Plot of complex permittivity $\left(\varepsilon_{r}\right)$ and conductivity $(\sigma)$ for real (dashed) and measured phantom (solid) human head tissue las

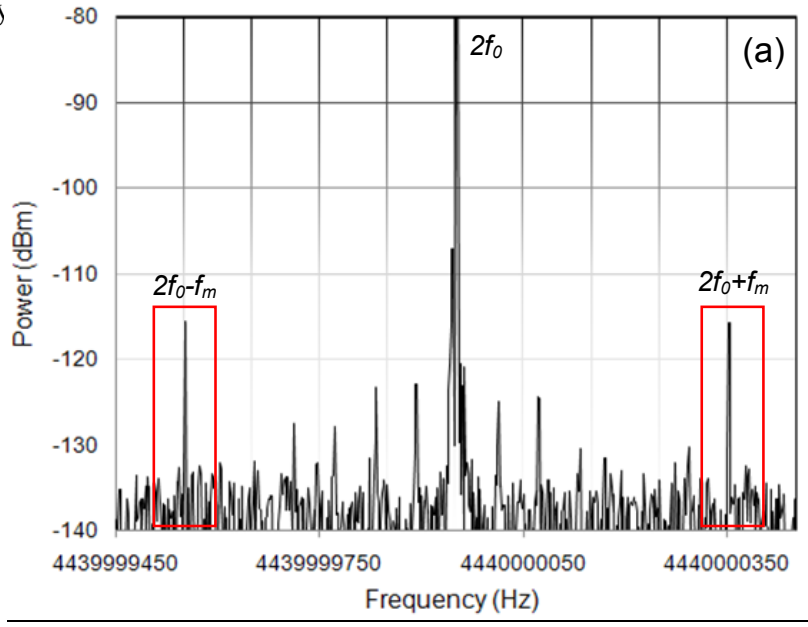

\begin{tabular}{|c|c|c|}
\hline SNR & Free Space & Phantom \\
\hline Low Resistivity Si & 0 & 0 \\
\hline High Resistivity Si & 38.9 & 22.0 \\
\hline Glass & 36.6 & 21.3 \\
\hline
\end{tabular}

Figure 5. (a) Spectral plot of wirelessly backscattered neuropotentials $\left(2 f_{0} \pm f_{m}\right.$, labeled) from microsystem in phantom $\left(V_{m}=50 \mathrm{mV}_{\mathrm{pp}}, f_{m}=400\right.$

$\mathrm{Hz}$ ). (b) SNR measurements for 3 different microsystem substrates.

\section{ACKNOWLEDGEMENT}

This work is supported in part by NSF (ECCS-0702227), NIH (5R21NS059815-02), and NASA Graduate Student Research Program (NNX09AK93H).

\section{REFERENCES}

[1] H.N. Schwerdt, W. Xu, S. Shekhar, A. Abbaspour-Tamijani, B.C. Towe, F.A. Miranda, and J. Chae, "A fully passive wireless microsystem for recording of neuropotentials using RF backscattering methods," IEEE J. MEMS, vol. 20, no. 5, pp. 1119-1130, Oct. 2011.

[2] A. Abbaspour-Tamijani, M. Farooqui, B.C. Towe, and J. Chae, "A miniature fully-passive microwave back-scattering device for shortrange telemetry of neural potentials," Proc. Ann. Int. Conf. IEEE-EMBS, Aug. 2008, pp. 129-132.

[3] K. Ito, K. Furuya, Y. Okano, and L. Hamada, "Development and characteristics of a biological tissue-equivalent phantom for microwaves." Electronics and Communications in Japan (Part I: Communications), vol. 84, pp. 67-77, 2001.

[4] C. Gabriel. "Compilation of the dielectric properties of body tissues at RF and microwave frequencies." Report N.AL/OE-TR- 1996-0037, Occupational and environmental health directorate, June 1996. 


\title{
Wireless Performance of a Fully Passive Neurorecording Microsystem Embedded in Dispersive Human Head Phantom
}

\author{
Helen N. Schwerdt ${ }^{1}$, Junseok Chae ${ }^{1}$, Félix A. Miranda ${ }^{2}$ \\ ${ }^{1}$ School of Electrical, Computer, and Energy Engineering \\ Arizona State University, Tempe, Arizona, USA \\ ${ }^{2}$ Antenna and Optical Systems Branch \\ NASA Glenn Research Center, Cleveland, Ohio, USA
}

2012 IEEE International Symposium on Antennas and Propagation Chicago, Illinois, USA July 9, 2012 (Session 156.2) 


\section{Outline}

- Introduction

- Operation

- Microsystem

- Wireless Backscattering

- Fabrication

- Wireless Performance in Air

- Preparation of Phantom Emulating Human Head

- Wireless Performance in Phantom

- Conclusion \& Future Work 


\section{Motivation}

"Neurorecording" $\rightarrow$ recording neuropotentials

(ie. electrical activity originating from neural signaling in brain or other nervous systems)

\begin{tabular}{|l|l|}
\hline Paraplegia/Paralysis & 6 (USA) \\
\hline Alzheimer's Disease & 5.3 (USA) \\
\hline Epilepsy & 4 (USA), 50 (world) \\
\hline Parkinson's Disease & 1 (USA) \\
\hline
\end{tabular}

BrainGate $\mathrm{BCl}^{3}$

- Prosthetics \& rehabilitation ${ }^{3-5}$

- Treatment

- Brain machine interfaces

- Advance understanding of brain

- One of the least understood and most important organs

- Most CNS knowledge based on conjecture

CNS = central nervous system

$\mathrm{BCl}=$ brain computer interface

$E E G=$ electroencephalography

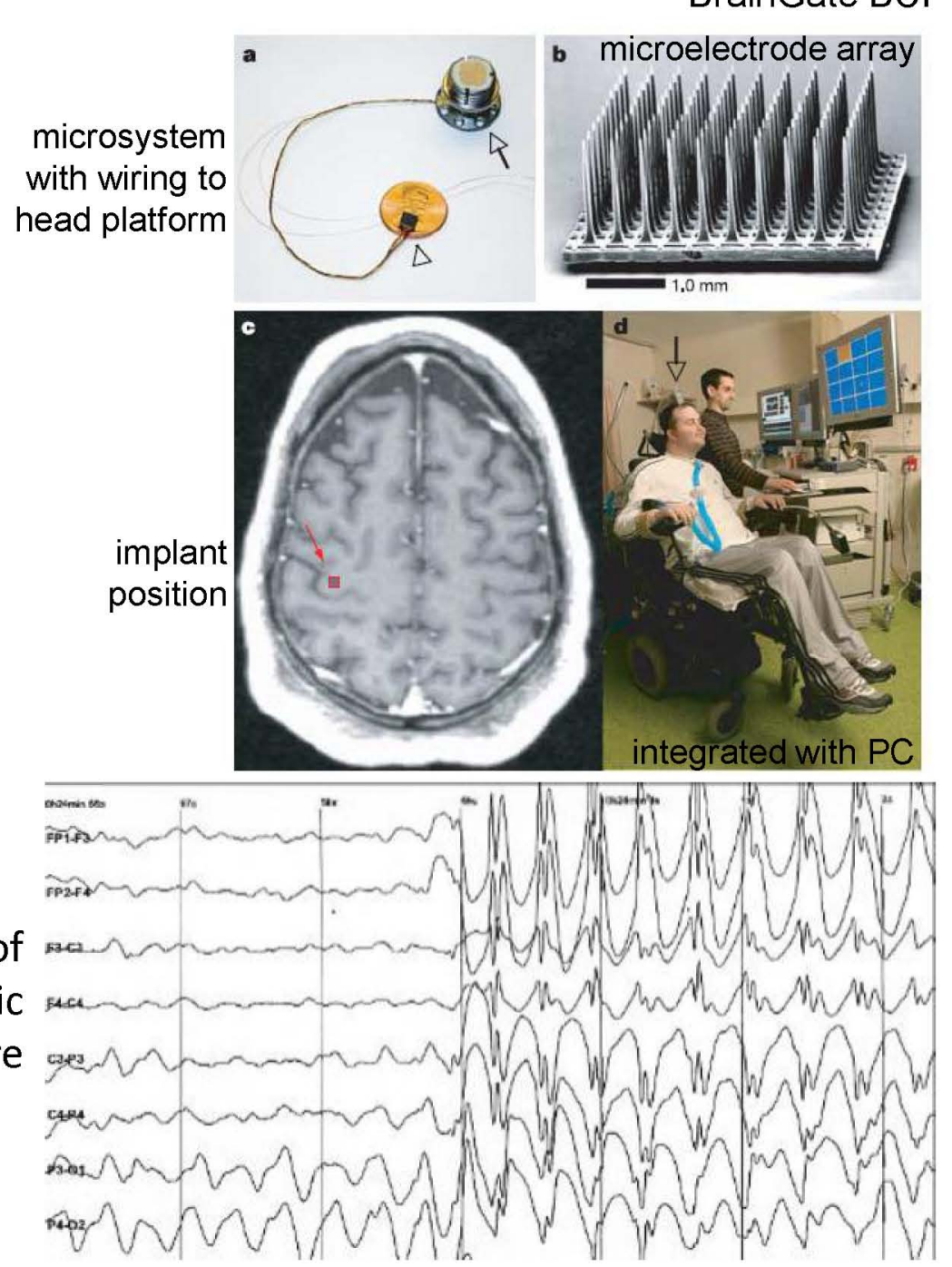




\section{Current Recording Systems \\ .}

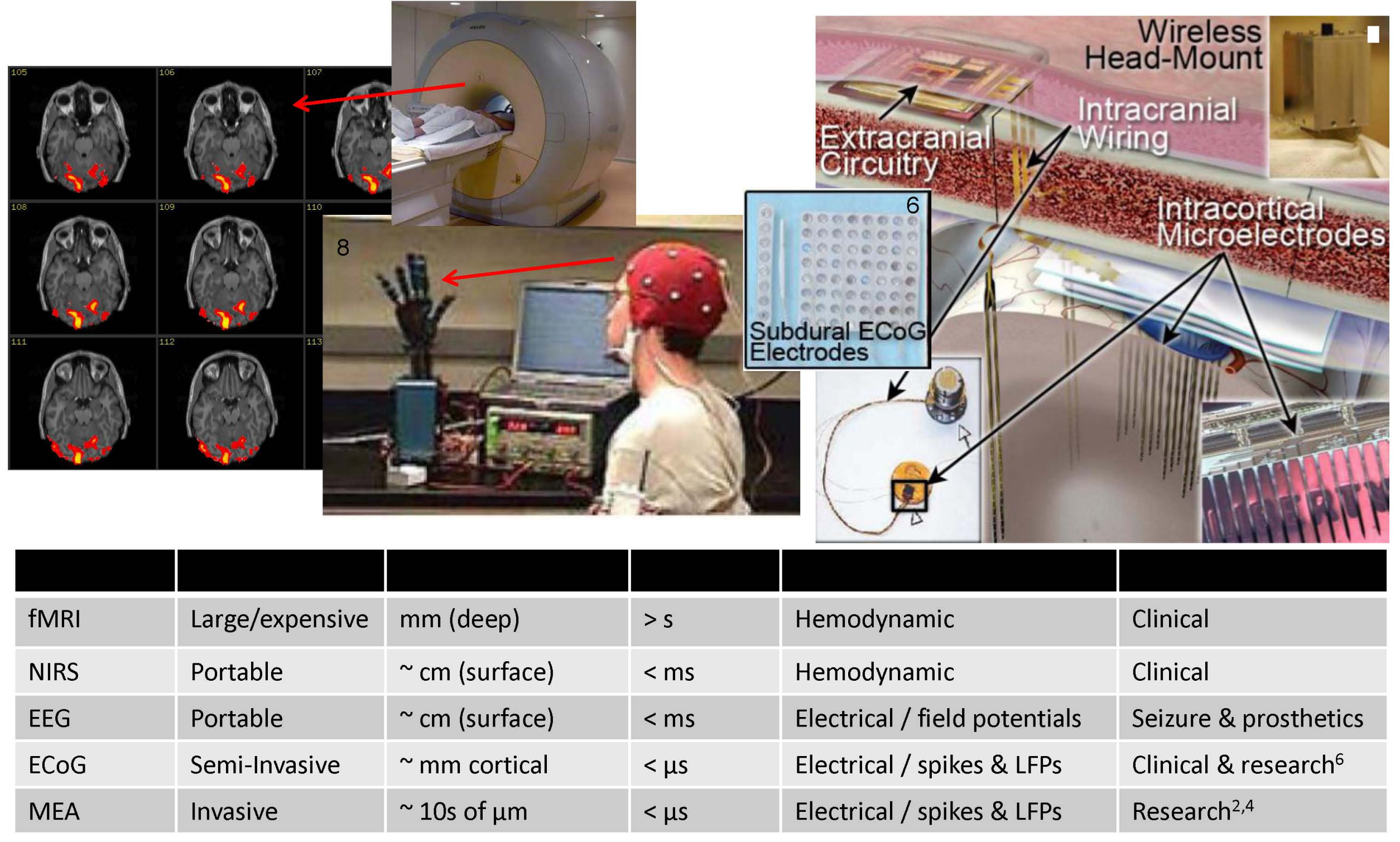

$f M R I=$ functional magnetic resonance imaging NIRS = near infrared spectroscopy

$E C O G$ = electrocorticography

$M E A=$ microelectrode array 


\section{Fully Passive Wireless Neurorecorder}

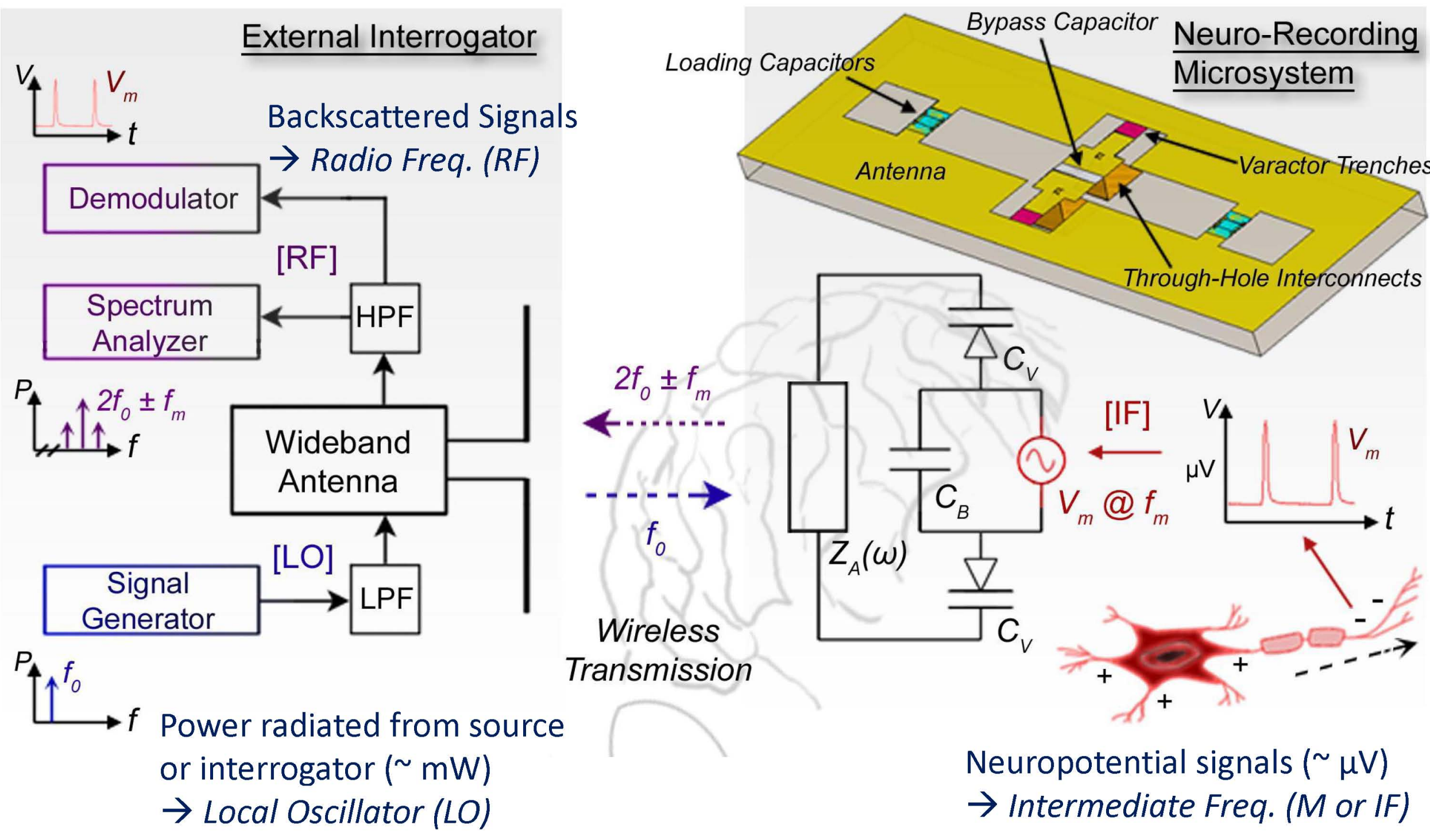




\section{Microsystem: Nonlinear Mixer}

Nonlinear Mixer $\rightarrow$ Passive Recording of Neuropotentials $\left(V_{m}\right)$

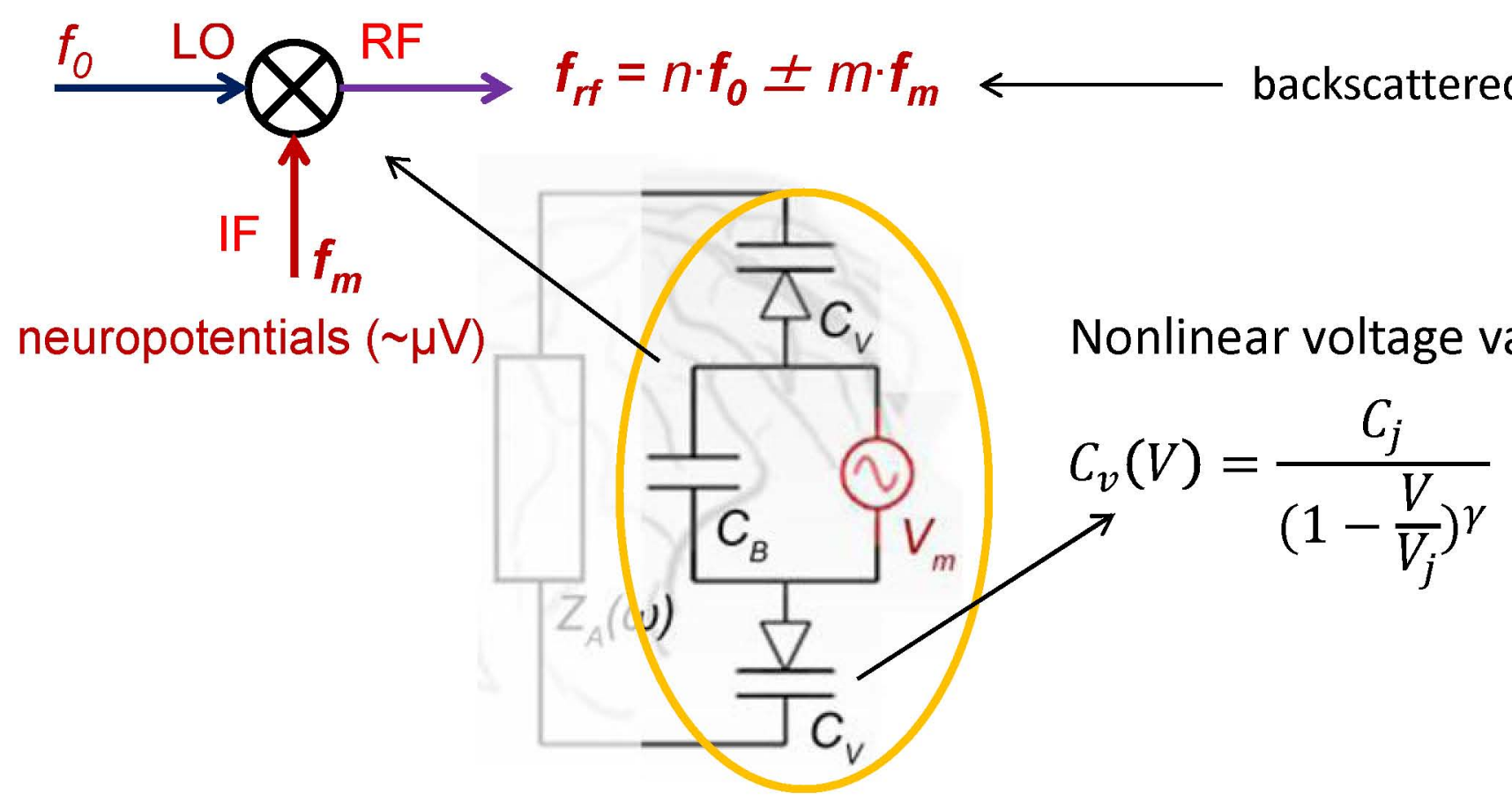

Mixer Output (Taylor Series Approximation):

$I=\left(c_{1 a}+c_{3 a}\right) \sin \left(\omega_{0} t\right)+\left(c_{1 a}+c_{3 a}\right) \sin \left(\omega_{m} t\right)+c_{3 b} \sin \left(2 \omega_{0} t \pm \omega_{m} t\right)+c_{3 b} \sin \left(\omega_{0} t \pm 2 \omega_{m} t\right)+\ldots$ 


\section{Microsystem: Integrated Antenna}

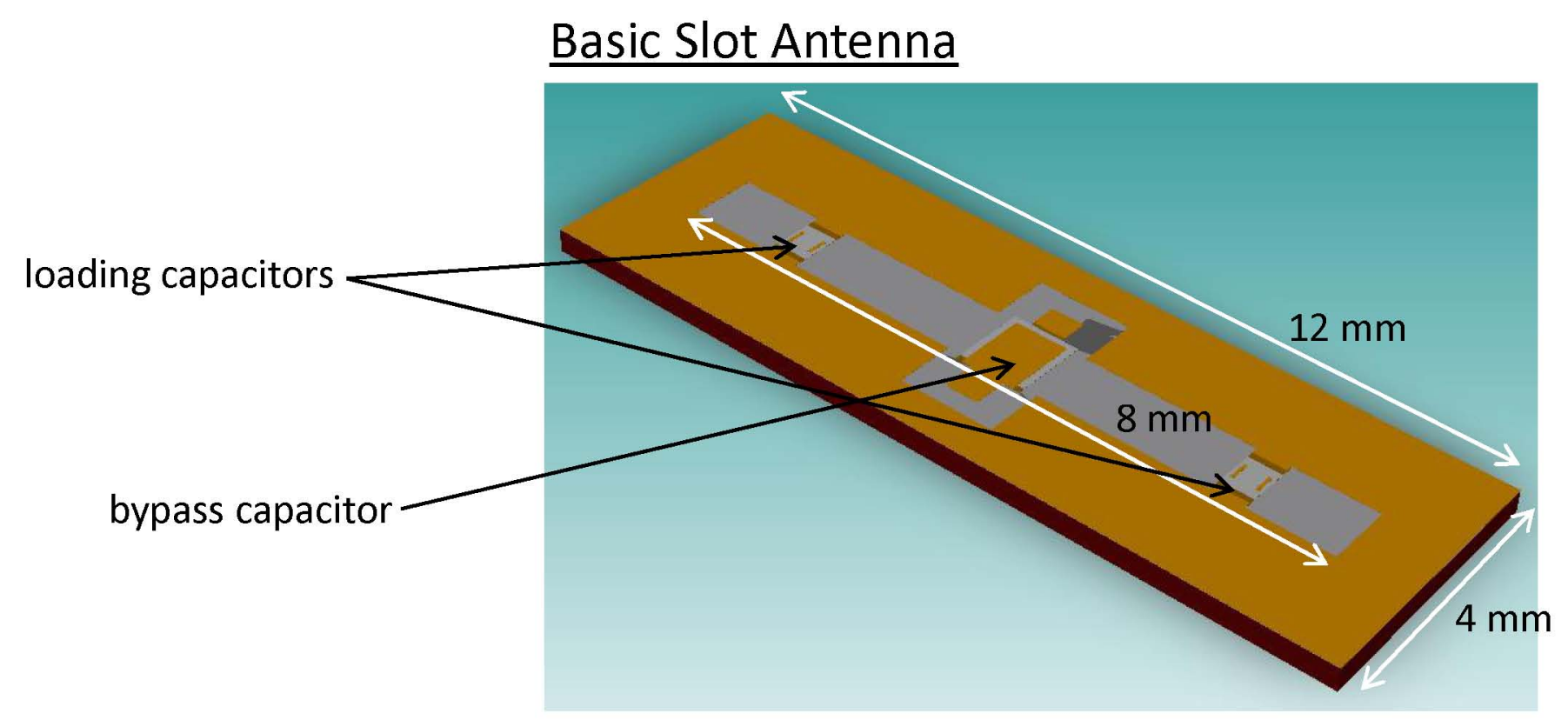

Electrically small antenna $\rightarrow$ antenna Gain limited to $\leq 1.76 \mathrm{dBi}$

Loading capacitors $\rightarrow$ tune frequency (dual-band operation @ $f_{0} \& 2 f_{0} \pm f_{m}$ ) 


\section{Wireless Backscattering System}

\section{Wireless Path Loss in Backscattering System}

External Interrogator

Neurorecording Microsystem

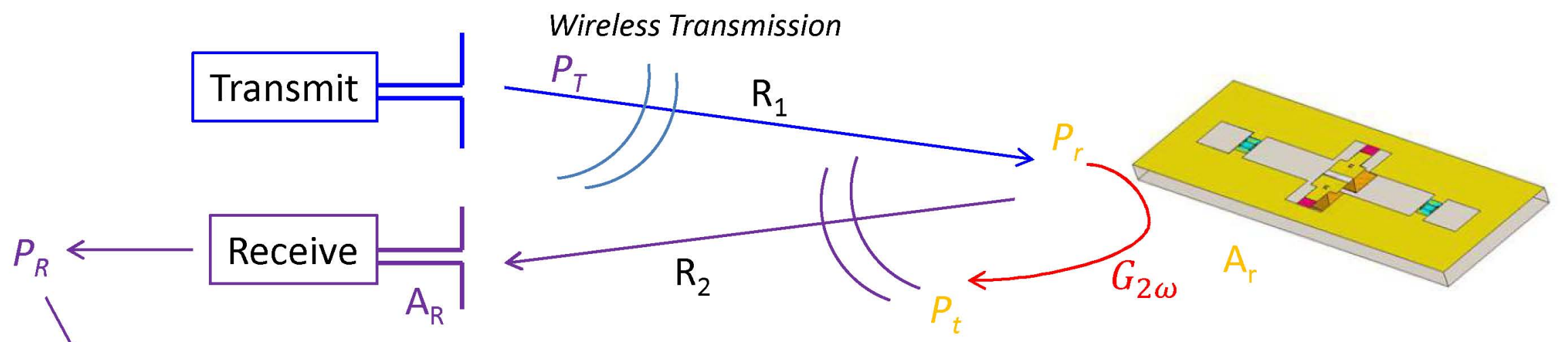

$\mathrm{P}_{\mathrm{IM} 3}\left(2 f_{0} \pm f_{m}\right)$ Backscattered:

$$
\begin{gathered}
P_{R}=\sigma \frac{P_{T} G_{T}}{4 \pi R_{1}^{2}} \cdot \frac{G_{t}}{4 \pi R_{2}^{2}} \cdot \frac{\lambda_{2}^{2}}{4 \pi} G_{R}=\sigma \frac{P_{T} G_{T} G_{t}}{(4 \pi)^{2} R^{4}} \cdot \frac{\lambda^{2}}{4 \pi} G_{R} \\
\left(R_{1}=R_{2}=R\right) \\
\sigma=4 \pi r^{2} \frac{S_{t}}{S_{r}}=\frac{\lambda_{1}^{2}}{4 \pi} G_{r} G_{2 \omega} G_{t}
\end{gathered}
$$

\begin{tabular}{|l|l|}
\hline$P_{T,} P_{R}$ & $\begin{array}{l}\text { Power transmitted/received by external } \\
\text { interrogator }\end{array}$ \\
\hline$G_{T}, G_{R}$ & $\begin{array}{l}\text { Gain of external interrogator transmit/receive } \\
\text { antenna modes }\end{array}$ \\
\hline$A_{r}, A_{R}$ & $\begin{array}{l}\text { Effective aperture of microsystem/external } \\
\text { interrogator antenna }\left(\lambda^{2} / 4 \pi\right)\end{array}$ \\
\hline$G_{2 \omega}$ & Conversion gain of onboard nonlinear mixer \\
\hline$G_{t}, G_{r}$ & $\begin{array}{l}\text { Gain of microsystem transmit/receive antenna } \\
\text { modes }\end{array}$ \\
\hline$\lambda_{1}, \lambda_{2}$ & $\begin{array}{l}\text { Wavelength at } f_{0} \text { (supply) and } 2 f_{0} \pm f_{m} \\
\text { (backscatter) frequencies }\end{array}$ \\
\hline
\end{tabular}




\section{Fabrication}

\section{$\underline{\text { Fabrication Process }}$}
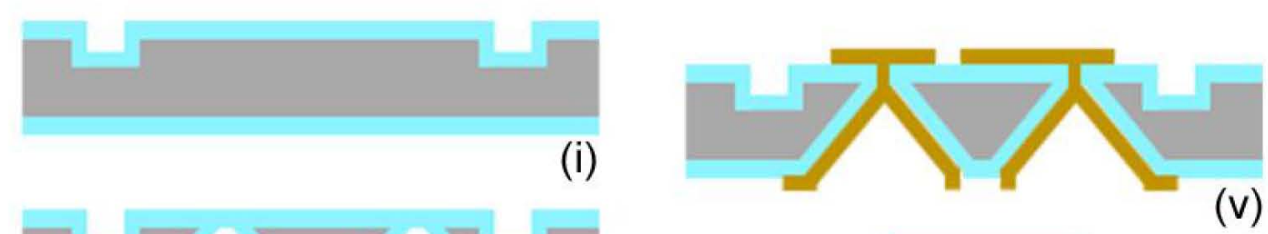

(ii)
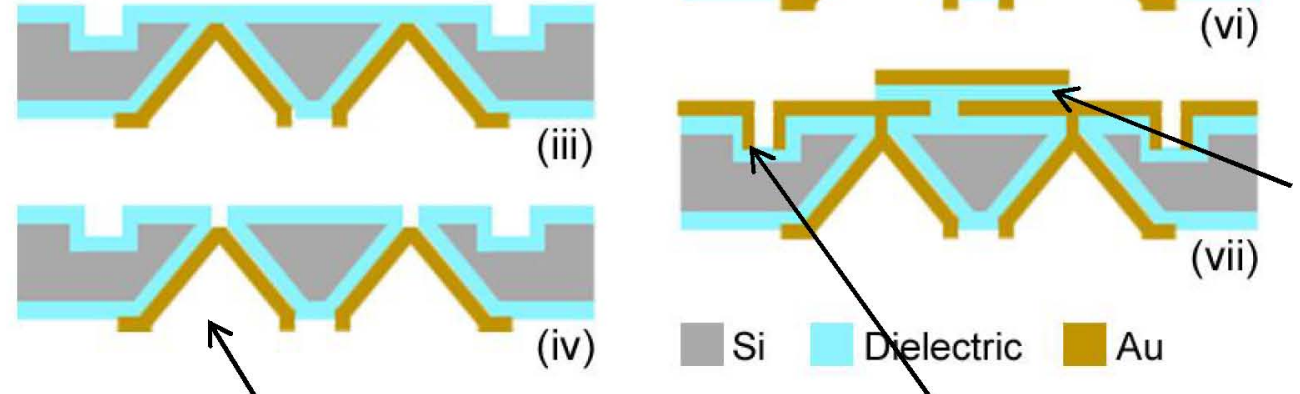

Completed Neurorecorder

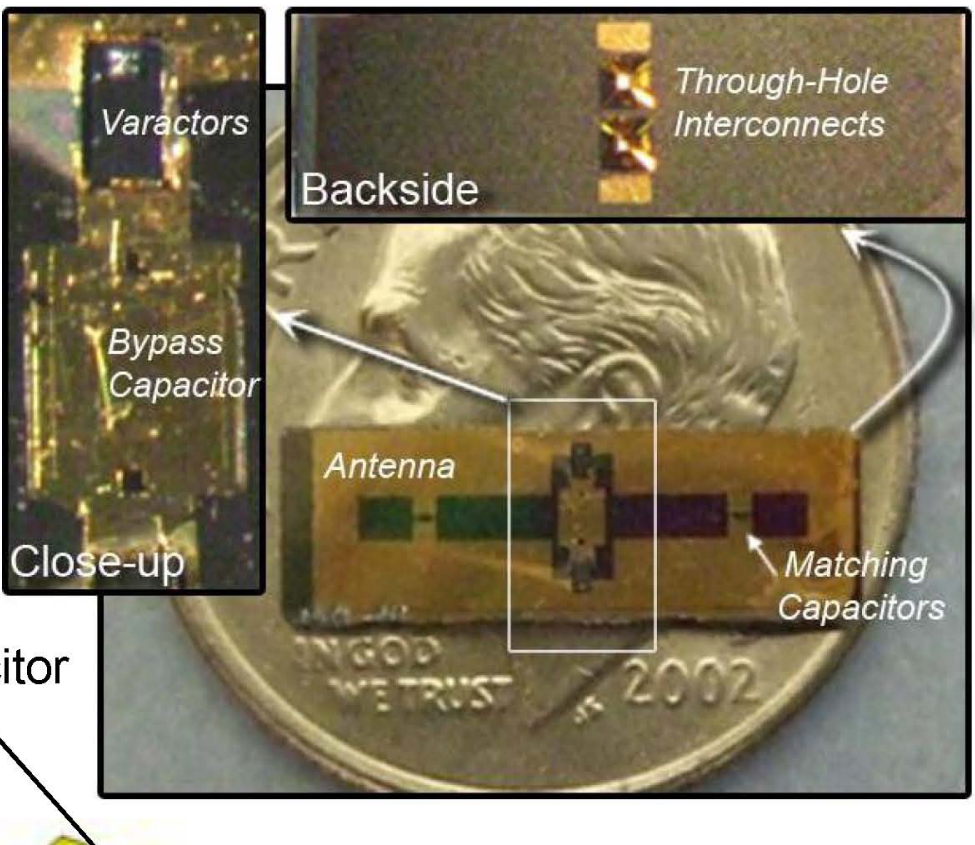

through-hole interconnects (contacts for neuropotential inputs)

capacitor
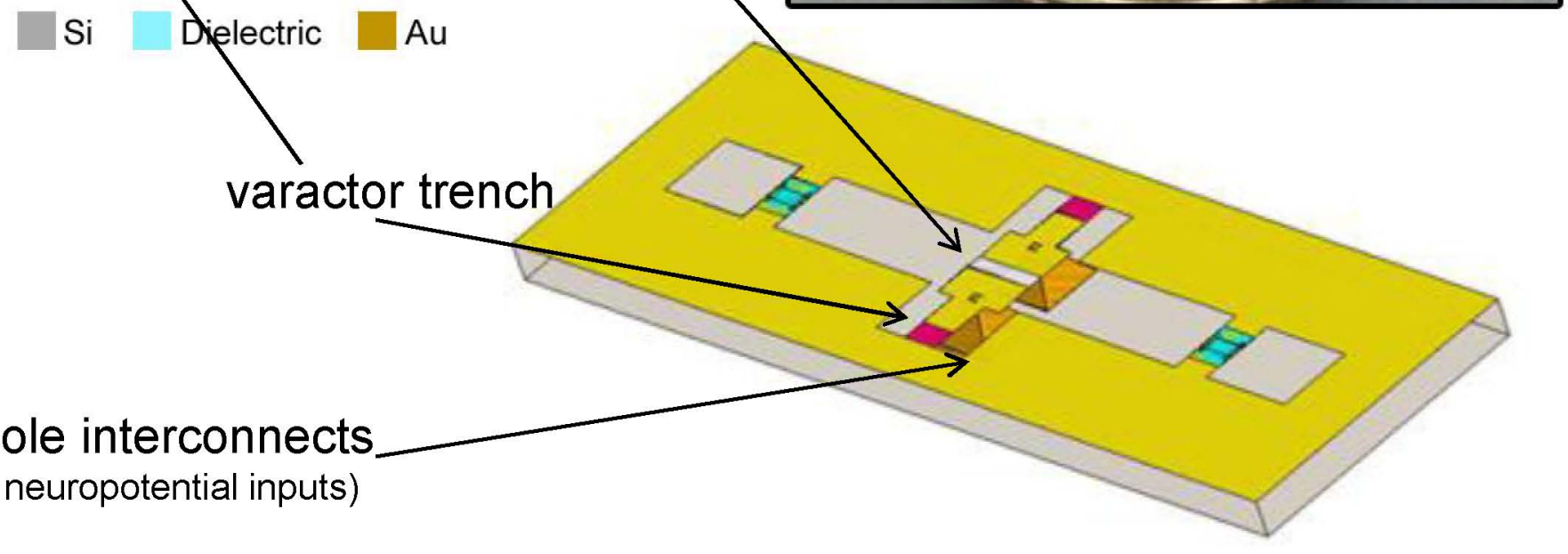


\section{Testing in Air}

Testing in "Free-Space" with emulated neuropotentials $\left(V_{m}\right)$ generated via function generator Measured wirelessly backscattered $3^{\text {rd }}$ order harmonics or $P_{I_{M 3}}\left(2 f_{0} \pm f_{m}\right)$

\section{Spectral Output}

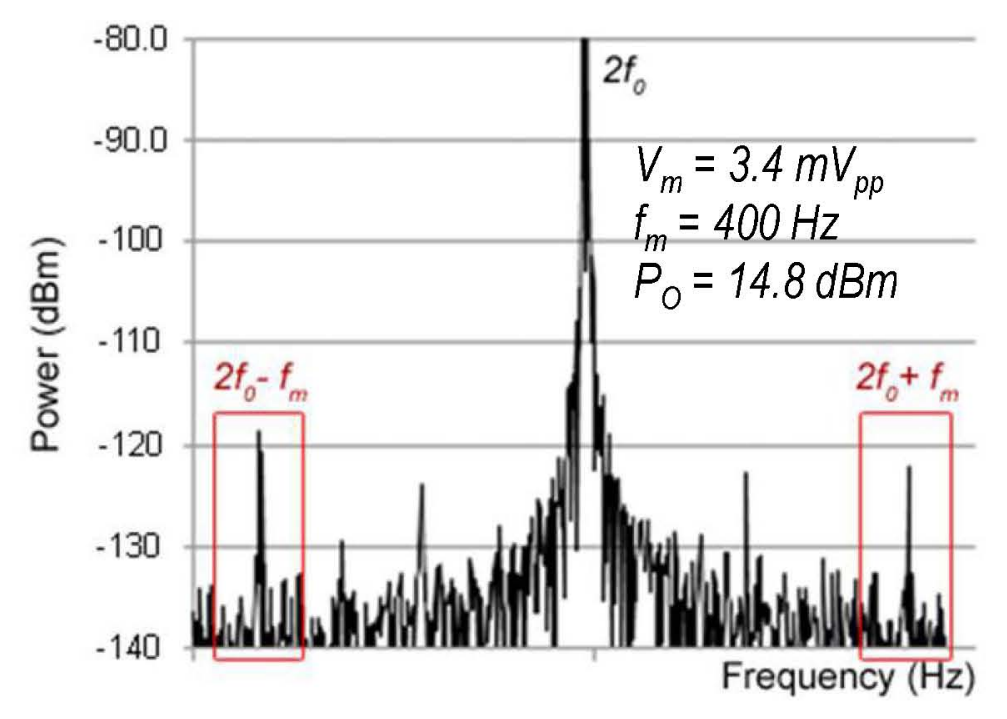

measured by spectrum analyzer
Demodulated Output

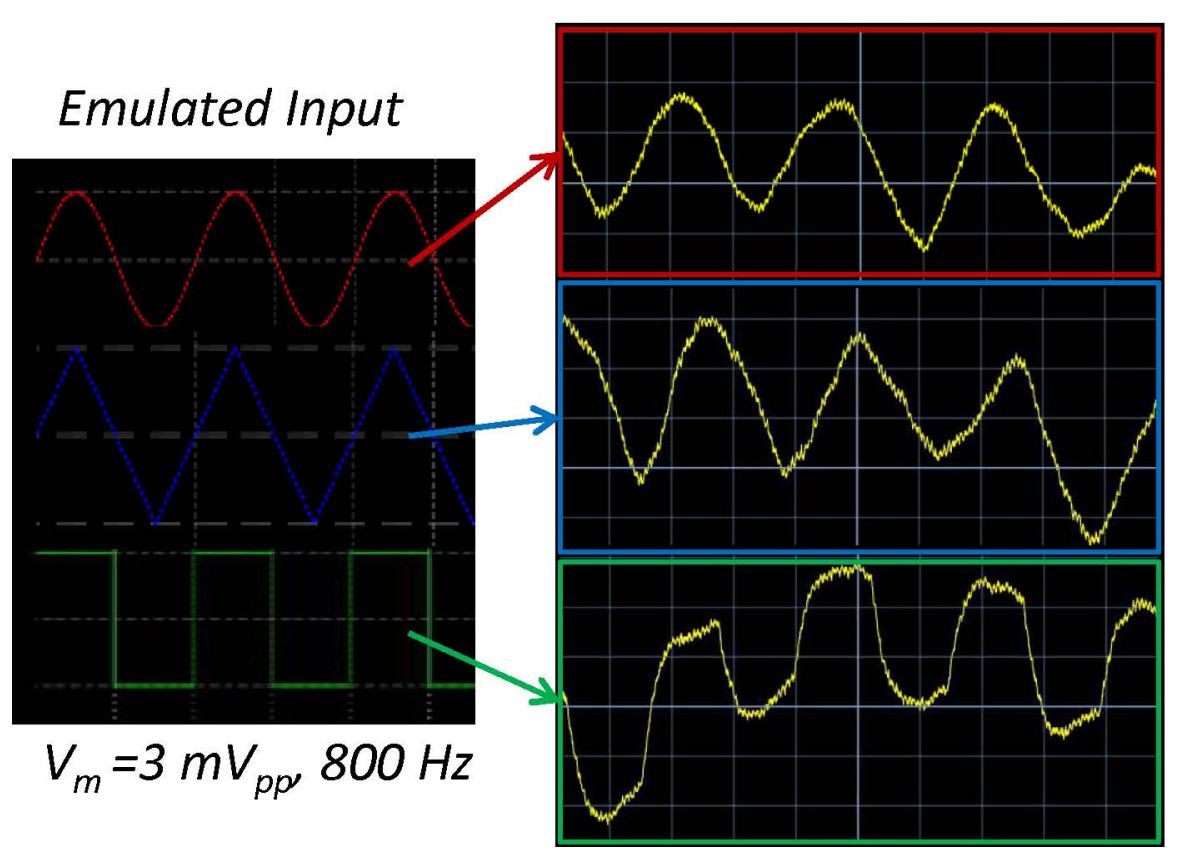

after synchronous AM demodulation 


\section{Testing with Frog}

Wireless Recording from Sciatic Nerve

Wired \& Wireless Measurements

Setup Procedure

Stimulate (inject current) higher end of nerve

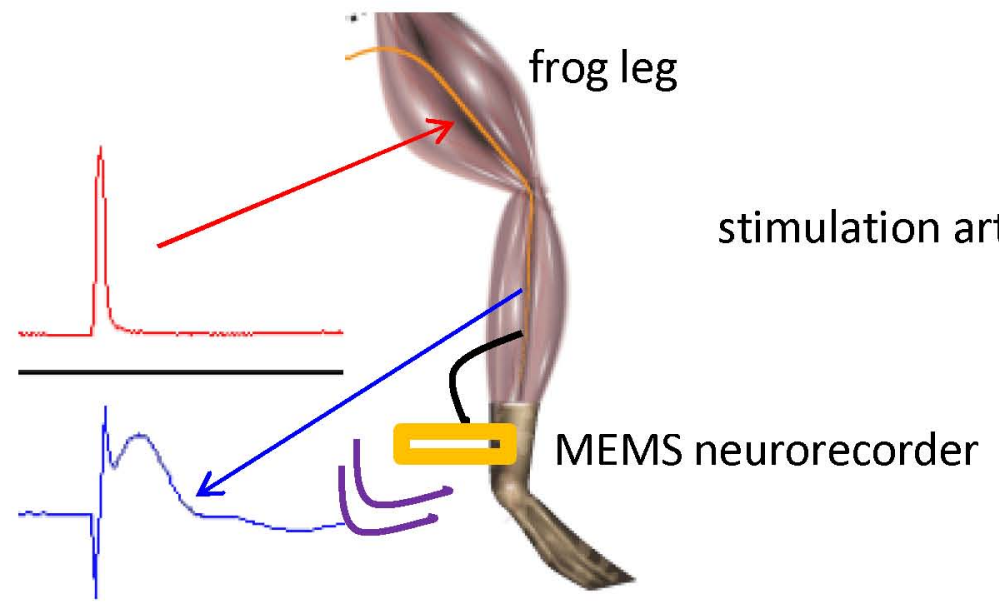

Wirelessly record CAPS

$$
\operatorname{CAPs}\left(V_{m}=500 \mu V_{p p} f_{m}=400 \mathrm{~Hz}\right)
$$
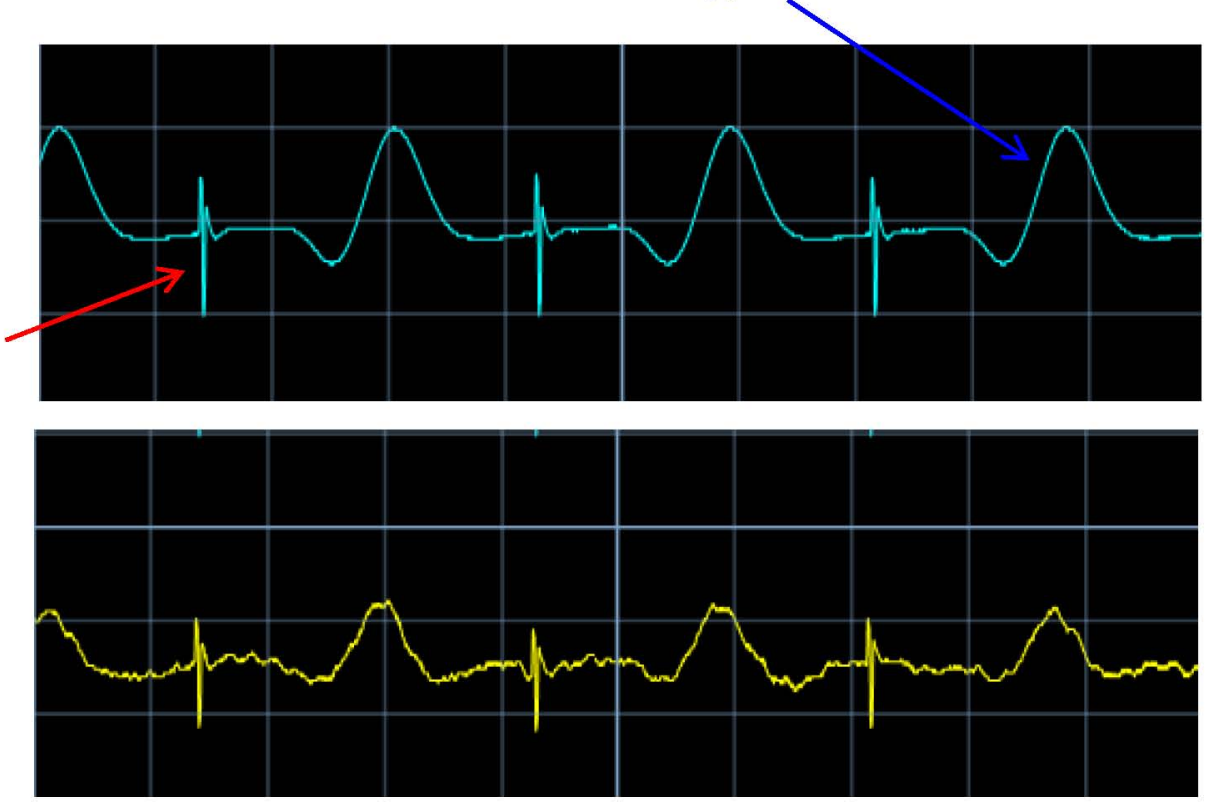

Used signal averaging on oscilloscope to improve SNR by around $11.3 \mathrm{~dB}$

CAPS = Compound Action Potentials (integration of many propagating action potentials/spikes in nerve bundle) 


\section{Summary of Measurements in Air}

$\begin{array}{lll}\text { Footprint } & \text { Dimensions } & 12 \times 4 \times 0.5 \mathrm{~mm}^{3} \\ \text { Detected Input } & \text { Bandwidth } & 5-3000 \mathrm{~Hz} \\ \text { Signals } & \text { Amplitude } & \geq 500 \mu \mathrm{V}_{\mathrm{pp}} \\ & \text { Distance } & \leq 1.5 \mathrm{~cm} \\ \text { RF } & \text { Radiated Power } & \leq 16.7 \mathrm{dBm}(47 \mathrm{~mW}) \\ \text { Transmission } & \text { Frequency } & 2.2-2.45 \mathrm{GHz} \\ \text { RF Reception } & \text { Sideband Level } & \leq-97 \mathrm{dBm}\left(\text { for } \mathrm{V}_{\mathrm{m}}=50 \mathrm{mV}_{\mathrm{pp}}\right) \\ & \text { Noise Floor } & \approx-136 \mathrm{dBm} \\ & \text { Frequency } & 4.4-4.9 \mathrm{GHz} \\ \text { Thermal } & \text { Temperature Rise } & \leq 0.15 \pm 0.1^{\circ} \mathrm{C} \\ \text { Characteristics } & \text { SAR (estimated) } & \leq 0.112 \mathrm{~W} / \mathrm{kg}\end{array}$




\section{Wireless Testing in Phantom Mimicking Real Head Tissues}

Final Goal - Implantable Wireless System in Lossy \& Inhomogeneous/Stratified Tissue Media

(until now wireless testing in air only)
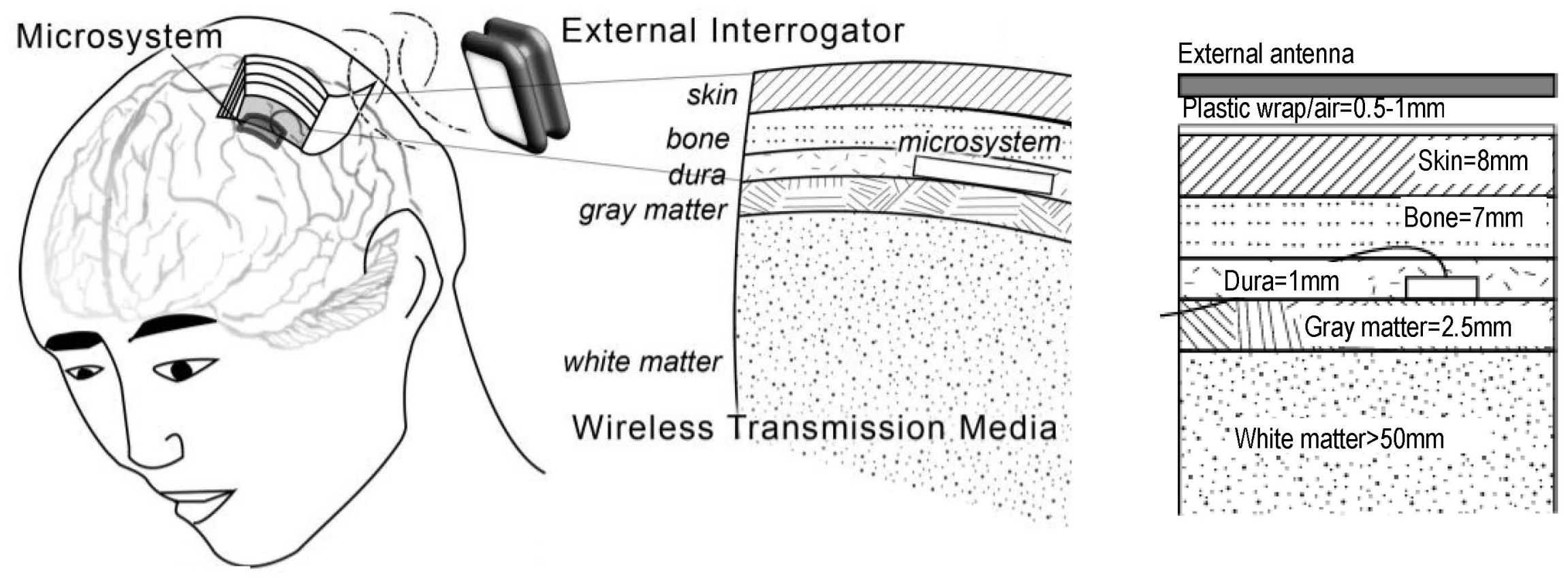


\section{Phantom Preparation}

Semisolid phantoms made for each head layer $^{23}$
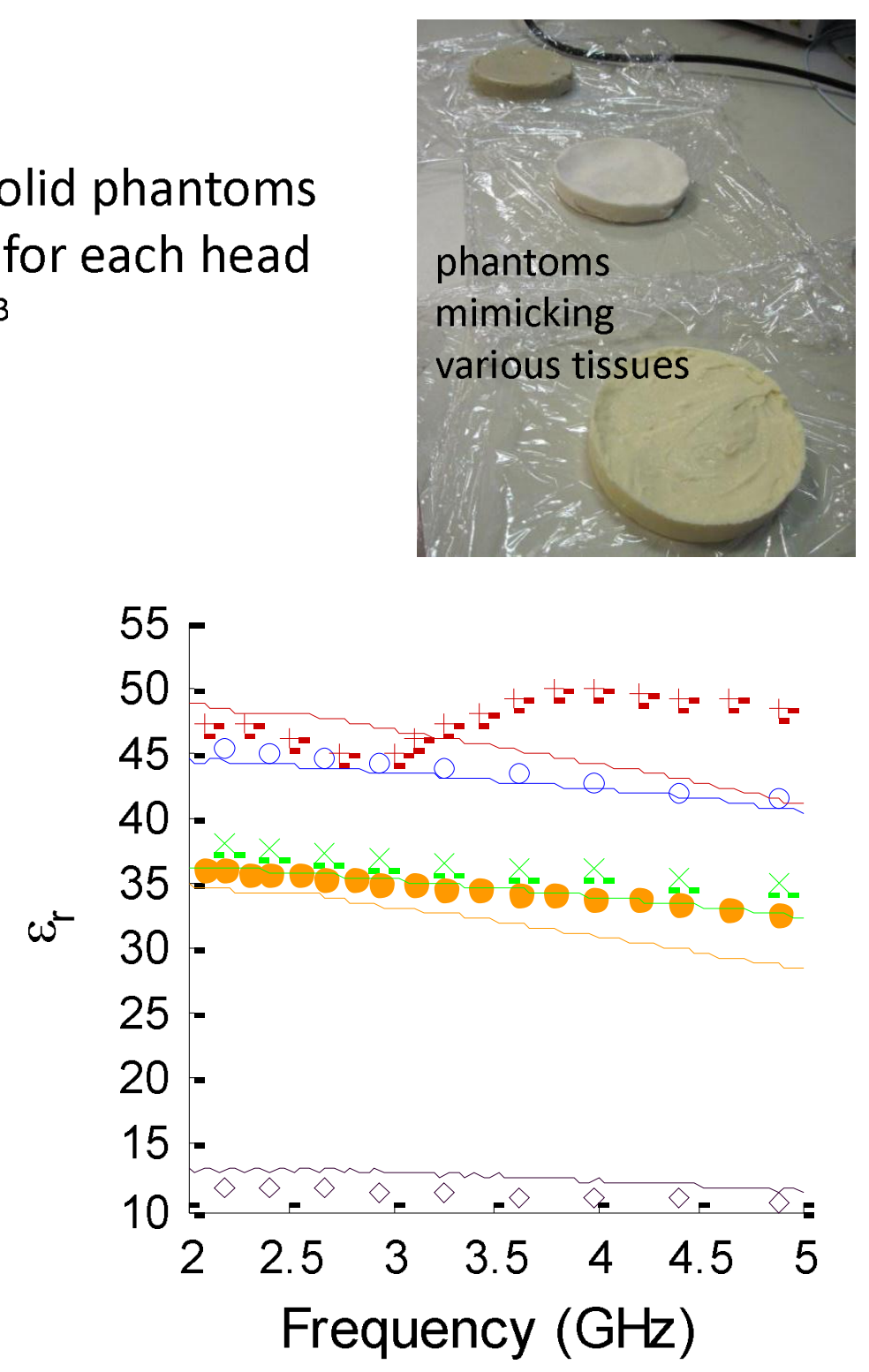

Measure dielectric properties
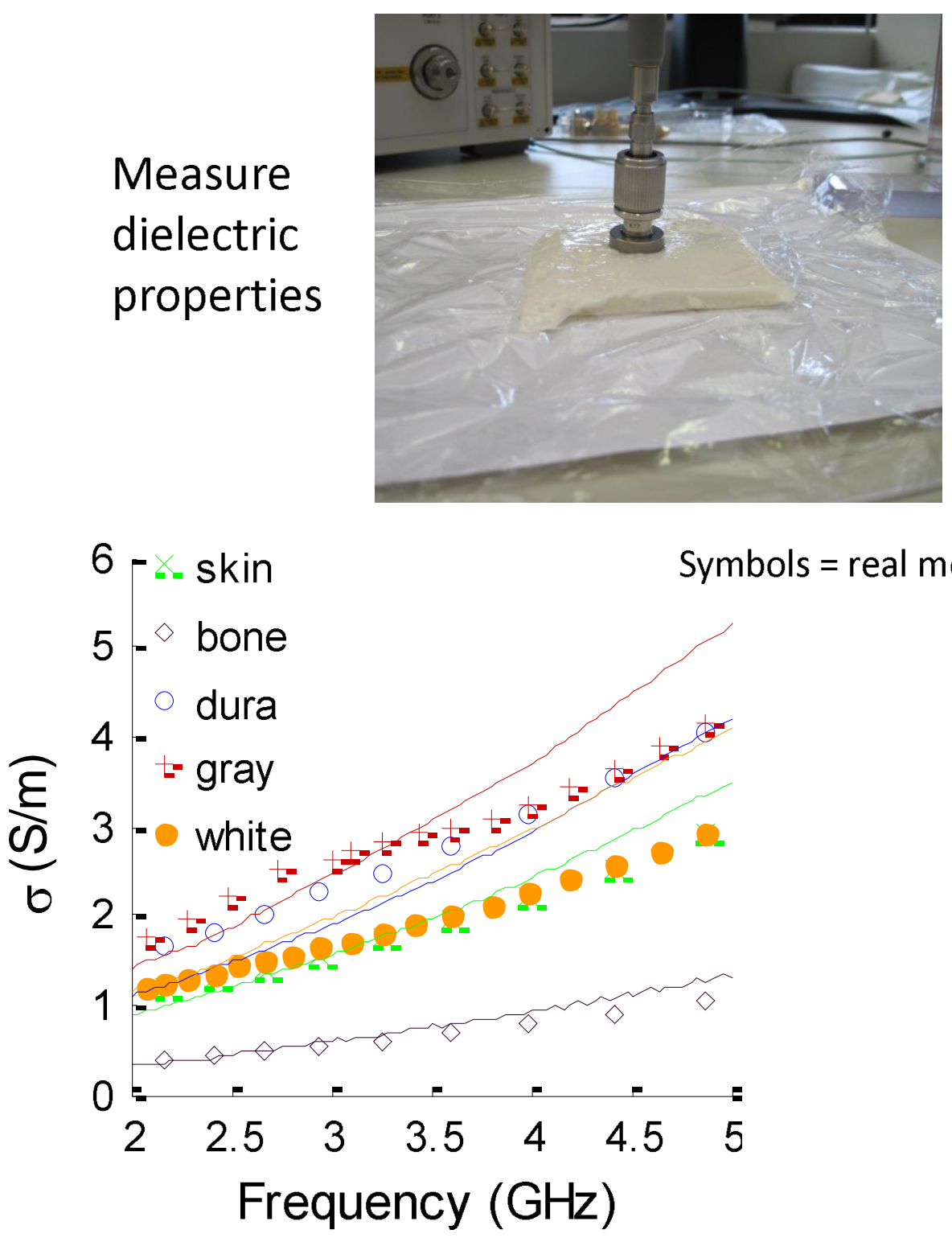


\section{Phantom Media Testing}

MEMS neuro-recorder (parylene coated)

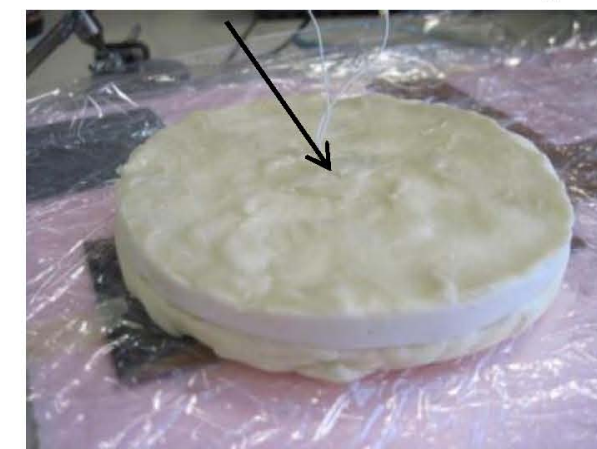

scalp/skull/dura

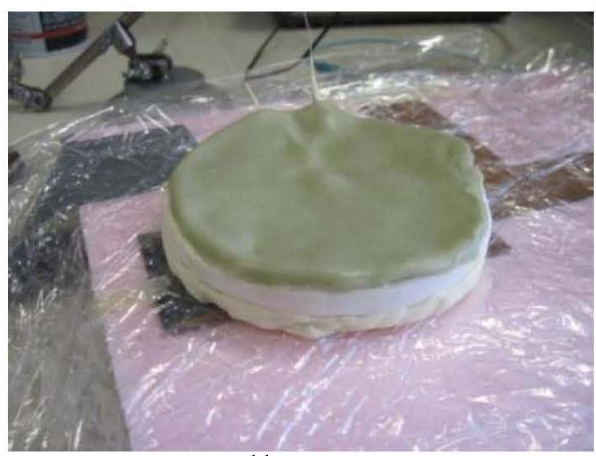

gray matter

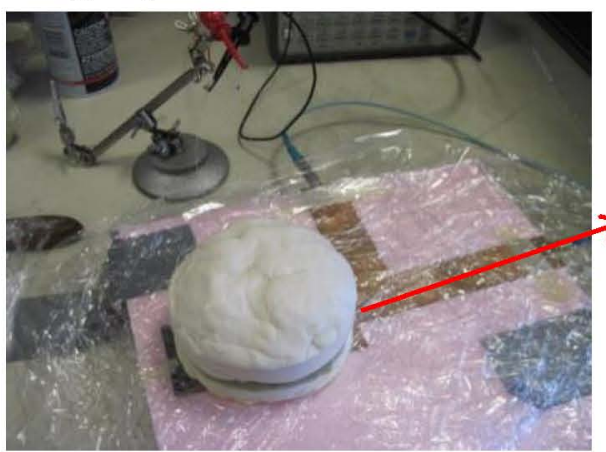

complete phantom
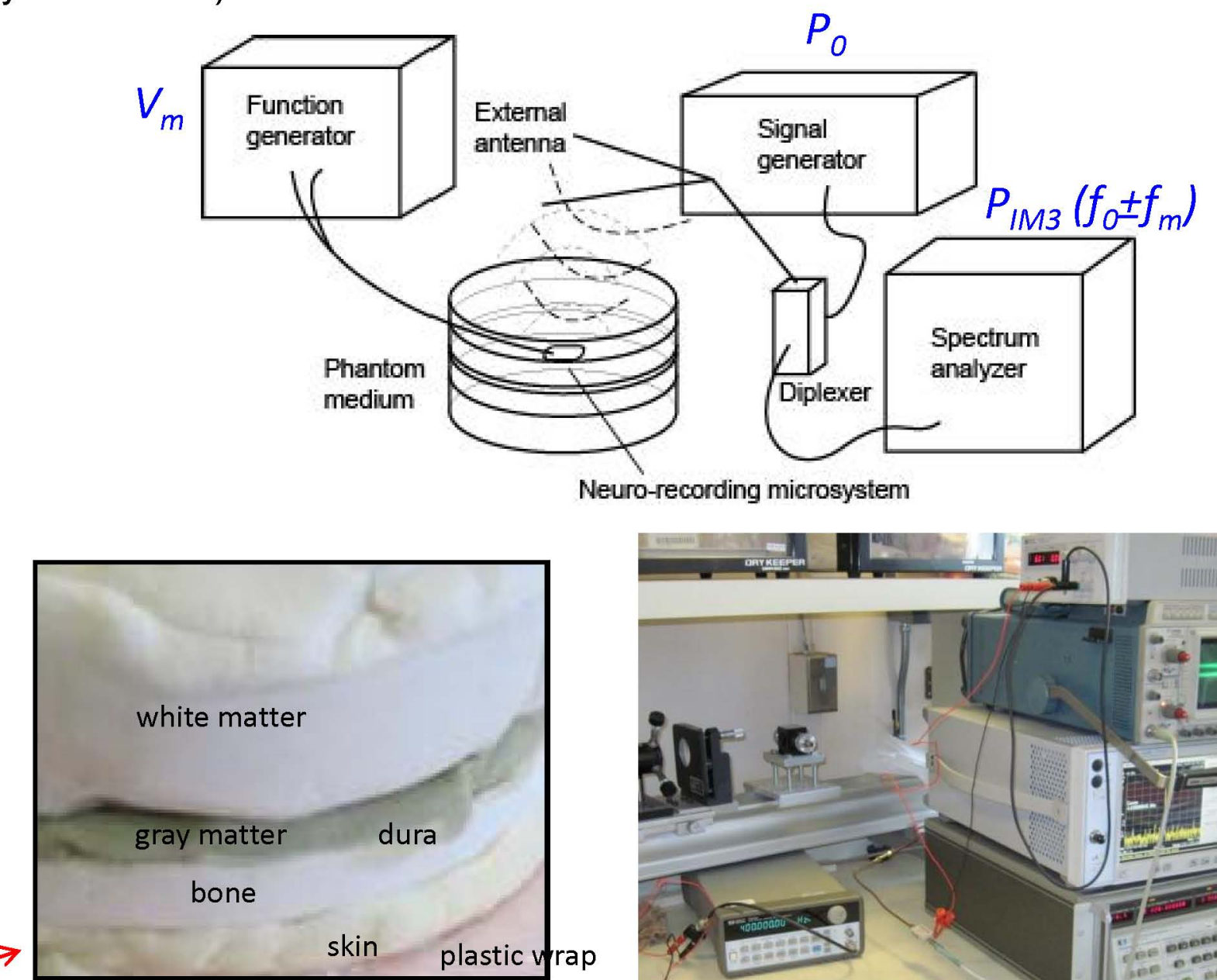

implant depth $1.5 \mathrm{~cm}$ (dura)

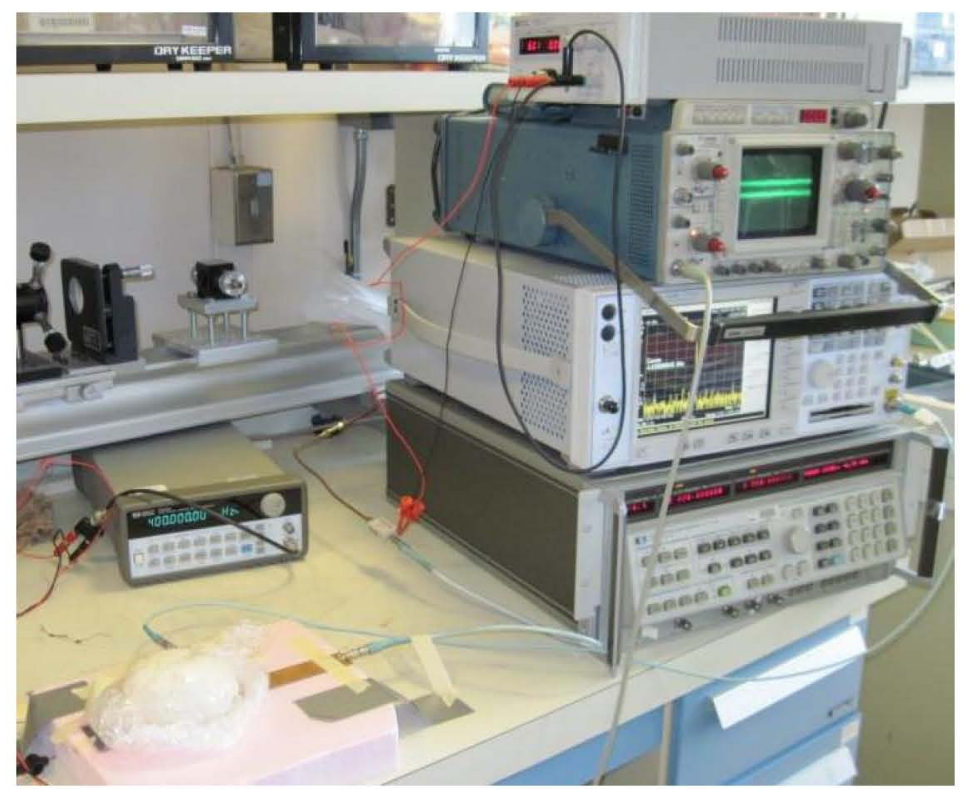




\section{Phantom Media Testing}

Measurements of Neurorecorders in Phantom

Spectral Output

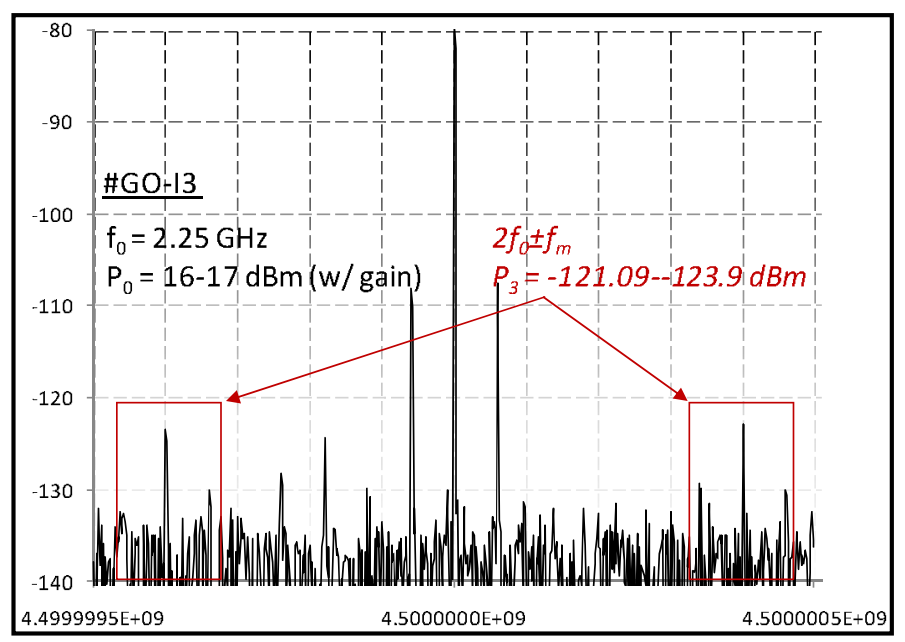

antenna

\begin{tabular}{|c|c|c|}
\hline \multicolumn{3}{|c|}{} \\
\hline Low Resistivity Si & 0 & $\mathbf{0}$ \\
\hline High Resistivity Si & 38.9 & $\mathbf{2 2 . 0}$ \\
\hline Glass & $\mathbf{3 6 . 6}$ & $\mathbf{2 1 . 3}$ \\
\hline
\end{tabular}

Skin depth accounts for real losses in phantom

$$
\delta=\frac{1}{\operatorname{Im}[\sqrt{\omega \mu(\omega \hat{\varepsilon}-j \sigma)}]}=\frac{1}{\operatorname{Im}\left[\sqrt{\omega^{2} \mu \varepsilon^{\prime}-j \omega \mu\left(\omega \varepsilon^{\prime \prime}+\sigma\right)}\right]}
$$

Comparison of glass \& silicon substrates
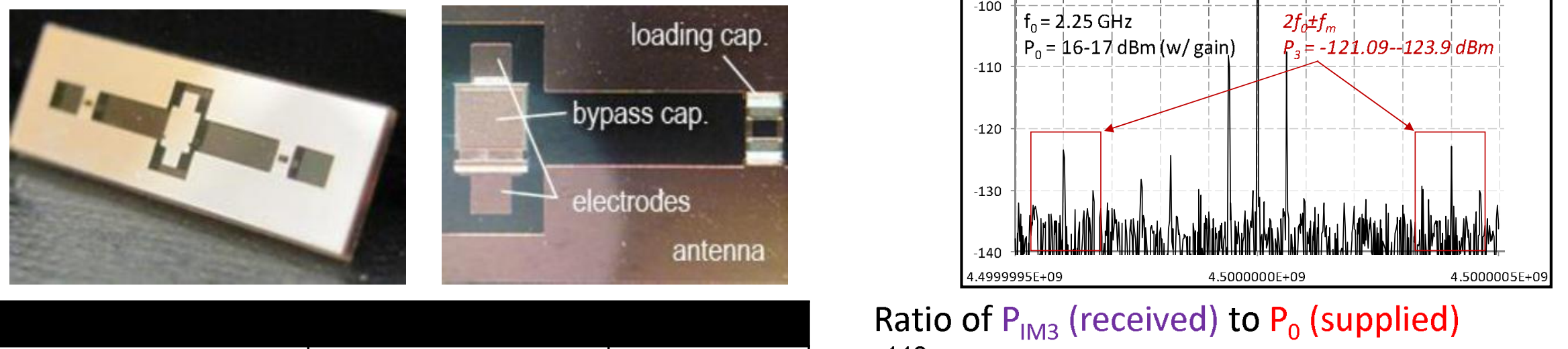
$-110$
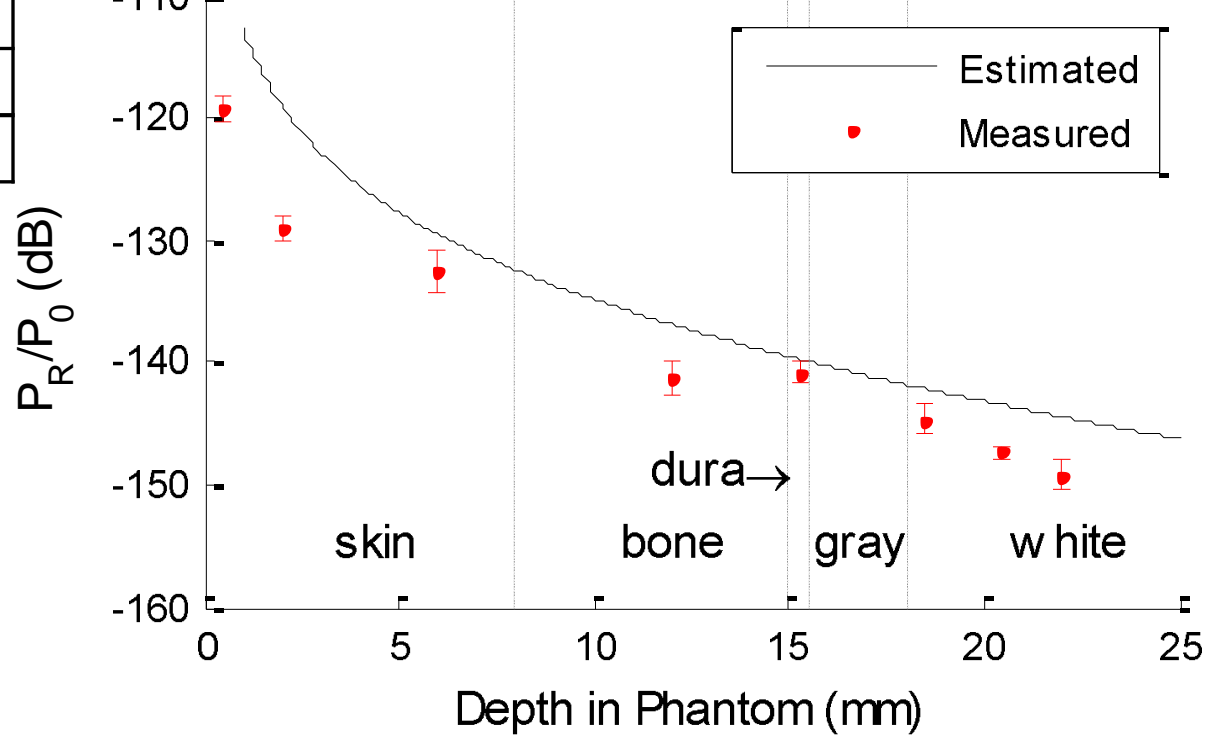


\section{Conclusion \& Future Work}

Conclusion:
\begin{tabular}{l|l|l|l}
\hline \multirow{2}{*}{ Detected $V_{m}$} & Amplitude & In Air & \\
\hline RF Transmission & Radiated Power & $\leq 500 \mu \mathrm{V}_{\mathrm{pp}}$ & \\
\cline { 2 - 4 } & Frequency & $2.2-2.45 \mathrm{dBm}(47 \mathrm{~mW})$ & \\
\hline RF Reception & $P_{\text {IM3 }}\left(V_{m}=50 m V_{p p}\right)$ & $\leq-97 \mathrm{dBm}$ & \\
\cline { 2 - 4 } & Noise Floor & $\approx-134 \mathrm{dBm}$ & \\
\hline \multirow{2}{*}{$\begin{array}{c}\text { Chermal } \\
\text { Chacteristics }\end{array}$} & Temperature Rise & $\leq 0.15 \pm 0.1^{\circ} \mathrm{C}$ & \\
\cline { 2 - 4 } & SAR & - & \\
\end{tabular}

Future Work:

-Thermal characterization of neurorecorder in phantom

-Increasing sensitivity (minimum detectable $V_{m}$ )

$\rightarrow$ Increasing nonlinearity $(\gamma)$ of varactors

$\rightarrow$ Increasing SNR of external interrogator (lowering phase noise) 


\section{References}

M.A.L. Nicolelis, Methods for Neural Ensemble Recordings. 2nd Ed. CRC Press, 2008.

A.V. Nurmikko, et al., "Listening to Brain Microcircuits for Interfacing With External World-Progress in Wireless Implantable Microelectronic Neuroengineering Devices," Proceedings of the IEEE 98(3), 375-388, March 2010.

L.R. Hochberg, et al., “Neuronal ensemble control of prosthetic devices by a human with tetraplegia," Nature 442, 164-171, 2006.

K.D. Wise, et al., "Microelectrodes, microelectronics, and implantable neural microsystems," IEEE Proceedings, vol. 96, no. 7, pp. 1184-1202 Jul. 2008.

D.R. Kipke, et al. “Advanced Neurotechnologies for Chronic Neural Interfaces: New Horizons and Clinical Opportunities," J. Neuroscience, 12, vol. 28, no. 46, 11830-11838, Nov. 2008.

J.P. Blount, et al., "Advances in intracranial monitoring," Neurosurg. Focus 25 (3):E18, 1-8, Sept. 2008.

R.R. Harrison, "Design of integrated circuits to observe brain activity," IEEE Proceedings, vol. 96, no.7, pp. 1203-1216, Jul. 2008.

M. Mollazadeh, et al. "Wireless Multichannel Acquisition of Neuropotentials," in Biomedical Circuits and Systems Conference, Nov. 2008 (BioCAS), 49-52.

Y.K. Song, et al., “Active microelectronic neurosensor arrays for implantable brain communication interfaces," IEEE Transactions in Neural Systems and Rehabilition Engineering, vol. 17, no. 4 , pp. 339-345, June 2009

C. Constantinescu. "Trends and Challenges in VLSI Circuit Reliability," IEEE Micro Magazine, 14-19, 2003.

Y. Li, et al. "Overcoming Early-Life Failure and Aging for Robust Systems," IEEE Design and Test of Computers, 28-39, 2009.

A. Waziri, et al., "Initial surgical experience with a dense cortical microarray in epileptic patients undergoing craniotomy for subdural electrode implantation," Neurosurgery, 64(3), 540-545, March 2009.

S. Kim et al., "Thermal impact of an active 3-d microelectrode array implanted in the brain," IEEE Trans. Neural Sys. Rehab. Eng., vol. 15, no. 4, pp. 493-501, 2007.

D. L. Means and K. W. Chan, "Evaluating compliance with FCC guidelines for human exposure to radiofrequency electromagnetic fields," FCC Office of Engineering and Technology OET, bulletin 65 , supplement $C, 2001$.

B.C. Towe, "Passive backscatter biotelemetry for neural interfacing," Proc. IEEE-EMBS Int. Conf. Neural Eng., May 2007, pp. 144-147.

A. Abbaspour-Tamijani, M. Farooqui, B.C. Towe, and J. Chae, "A miniature fully-passive microwave back-scattering device for short-range telemetry of neural potentials," Proc. Ann. Int. Conf. IEEE-EMBS, Aug. 2008, pp. 129-132.

C.A. Balanis, Advanced Engineering Electromagnetics. Hoboken, NJ: John Wiley \& Sons, 1989

J. Galejs, Antennas in Inhomogeneous Media. London, Great Britain: Pergamon Press, 1969

S.A. Maas, Nonlinear Microwave and RF Circuits. Norwood, MA: Artech House, 2003

C.A. Balanis, Antenna Theory: Analysis and Design. Hoboken, NJ: John Wiley \& Sons, 2005.

H.A. Wheeler, "Fundamental limitations of small antennas," IEEE Proceedings of the Institute of Radio Engineers, pp. 1479-1484, March 1947.

R.F. Harrington, "Effect of antenna size on gain, bandwidth, and efficiency," Journal of Research of the National Bureau of Standards-D, Radio Propagation, vol. 64D, no. 1, pp. 1-12, Jan. 1960,

K. Ito, K. Furuya, Y. Okano, and L. Hamada, "Development and characteristics of a biological tissue-equivalent phantom for microwaves." Electronics and Communications in Japan (Part I: Communications), vol. 84, pp. 67-77, 2001.

C. Gabriel, and S. Gabriel, "Compilation of the dielectric properties of body tissues at RF and microwave frequencies," Report for Armstrong Laboratory (AFMC), Occupational and Environmental Health Directorate, Radiofrequency Radiation Division: AL/OE-TR-1996-0037, TX, USA, 1996.

K.S. Cole, and R.H. Cole, "Dispersion and absorption in dielectrics," Journal of Chemical Physics, vol. 9, pp. 341-52, Apr. 1941.

D. L. Means and K. W. Chan, "Evaluating compliance with FCC guidelines for human exposure to radiofrequency electromagnetic fields," FCC Office of Engineering and Technology OET, bulletin 65 , supplement C, 2001.

J.C. LaManna, et al., "Stimulus-Activated Changes in Brain Tissue Temperature in the Anesthetized Rat", Metabolic Brain Disease, Vol. 4, pp. 225-237, 1989.

T. Fujii and Y. Ibata, "Effects of heating on electrical activities of guinea pig olfactory cortical slices," Eur. J. Physiol., vol. 392, pp. 257-260, 1982.

M. Ueda, J. Bures, and J. Fischer, "Spreading depression elicited by thermal effects of ultrasonic irradiation of cerebral cortex in rats," J. Neurobiol., vol. 8, pp. 381-393, 1977.

H.N. Schwerdt, W. Xu, S. Shekhar, A. Abbaspour-Tamijani, B.C. Towe, F.A. Miranda, and J. Chae, "A fully passive wireless microsystem for recording of neuropotentials using RF backscattering methods," IEEE J. MEMS, vol. 20, no. 5, pp. 1119-1130, Oct. 2011.

H.N. Schwerdt, F.A. Miranda, and J. Chae, "A Fully Passive Wireless Backscattering Neuro-Recording Microsystem Embedded in Dispersive Human Head Phantom Medium," IEEE Electron Device Letters, vol. 33, no. 6, pp. 908-910, June 2012. 


\section{Acknowledgement}

This work was supported by:

National Science Foundation (NSF) (\#ECCS-0702227)

National Institutes of Health (NIH) (\#5R21NS059815-02)

NASA Graduate Student Research Program (GSRP) Fellowship (\#NNX09AK93H) 


\section{Appendix I: Thermal Impact}

Fully Passive Wireless Microsystem $\Delta \mathrm{T}$ vs Wireless RF $P_{0}$ Supplied (in air \& @2 $25^{\circ} \mathrm{C}$ )

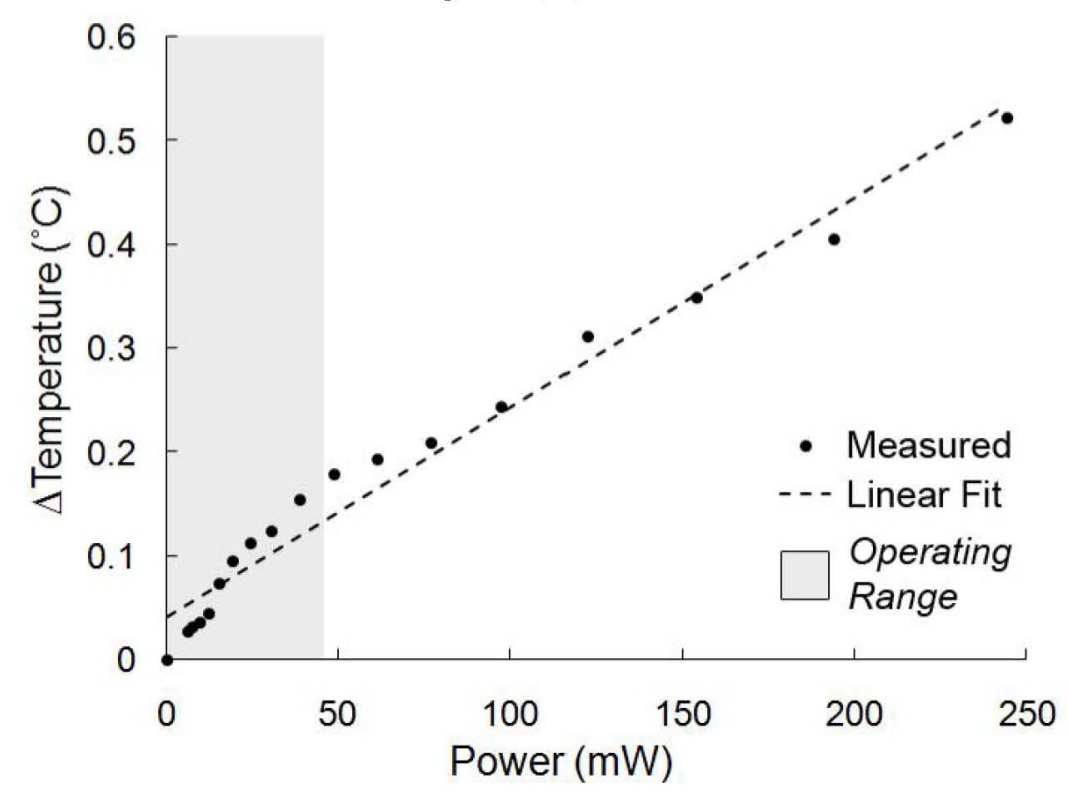

Utah Electrode Array $\Delta T$ vs P Dissipated

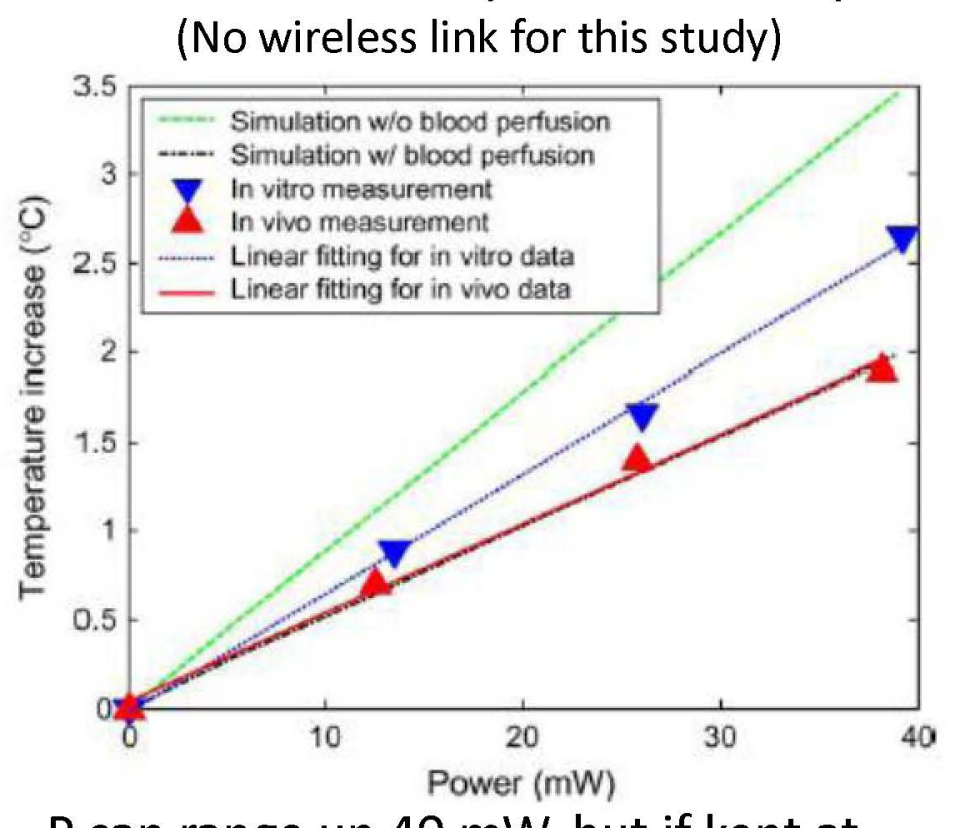

P can range up $40 \mathrm{~mW}$, but if kept at $13 \mathrm{~mW}, \Delta \mathrm{T}=0.38^{\circ} \mathrm{C}$

$\Delta \mathrm{T}>1{ }^{\circ} \mathrm{C} \rightarrow$ long-term effects on the brain tissue [20].

$\Delta \mathrm{T}>2{ }^{\circ} \mathrm{C} \rightarrow$ aberrant activity in brain (as shown in guinea pig olfactory cortical slices) [21] $\Delta \mathrm{T}>3{ }^{\circ} \mathrm{C} \rightarrow$ physiological abnormalities and tissue death (cortical spreading depression was observed by heating the cortex of anesthetized rats by $3.4^{\circ} \mathrm{C}$ ) [22] 


\section{Appendix II(a): Varactor Mixer}

$$
C(V)=\frac{C_{j}}{\left(1-\frac{V}{V_{j}}\right)^{\gamma}} \quad \begin{aligned}
& \text { Input: } \\
& V=V_{0} \cos \left(\omega_{0} t\right)+V_{m} \cos \left(\omega_{m} t\right)
\end{aligned}
$$

Output (Taylor Series Approx.): $\quad I=\frac{d}{d t}\left(\sum_{n=1}^{\infty} c_{n} V^{n}\right) V \quad I=\frac{d}{d t}\left(c_{1}+c_{2} V+c_{3} V^{2}+\ldots\right) V$

$$
I=\left(c_{1 a}+c_{3 a}\right) \sin \left(\omega_{0} t\right)+\left(c_{1 a}+c_{3 a}\right) \sin \left(\omega_{m} t\right)+
$$

$c_{2 a 1} \sin \left(2 \omega_{0} t\right)+c_{2 b} \sin \left(2 \omega_{m} t\right)+c_{2 c 1} \sin \left(\omega_{0} t \pm \omega_{m} t\right)+$

$c_{3 b} \sin \left(2 \omega_{0} t \pm \omega_{m} t\right)+c_{3 b} \sin \left(\omega_{0} t \pm 2 \omega_{m} t\right)+\ldots$

Targeted backscattered signals

$$
c_{3 b}=\frac{-C_{j}\left(\gamma^{2}+\gamma\right)}{V_{j}^{2}} \cdot\left(\frac{V_{0}^{2} V_{m}\left(\omega_{m}\right)}{4}+\frac{V_{0} V_{m}\left(V_{0} \omega_{0}+V_{m} \omega_{m}\right)}{2}\right)
$$

Back to Back Config. : $I=\frac{d}{d t}\left(\sum_{n=1}^{\infty} c_{n} V_{+}^{n+1}-c_{n} V_{-}^{n+1}\right)$

$$
\begin{gathered}
I=\frac{d}{d t}\left(c_{1} V_{+}-c_{1} V_{-}+c_{2} V_{+}^{2}-c_{2} V_{-}^{2}+c_{3} V_{+}^{3}-c_{3} V_{-}^{3}+\cdots\right) \\
I=\left(c_{1 a}+c_{3 a}\right) \sin \left(\omega_{0} t\right)+\left(c_{1 a}+c_{3 a}\right) \sin \left(\omega_{m} t\right)+c_{3 b} \sin \left(2 \omega_{0} t\right. \\
\left. \pm \omega_{m} t\right)+c_{3 b} \sin \left(\omega_{0} t \pm 2 \omega_{m} t\right)+\ldots
\end{gathered}
$$




\section{Appendix II(b): Varactor Mixer}

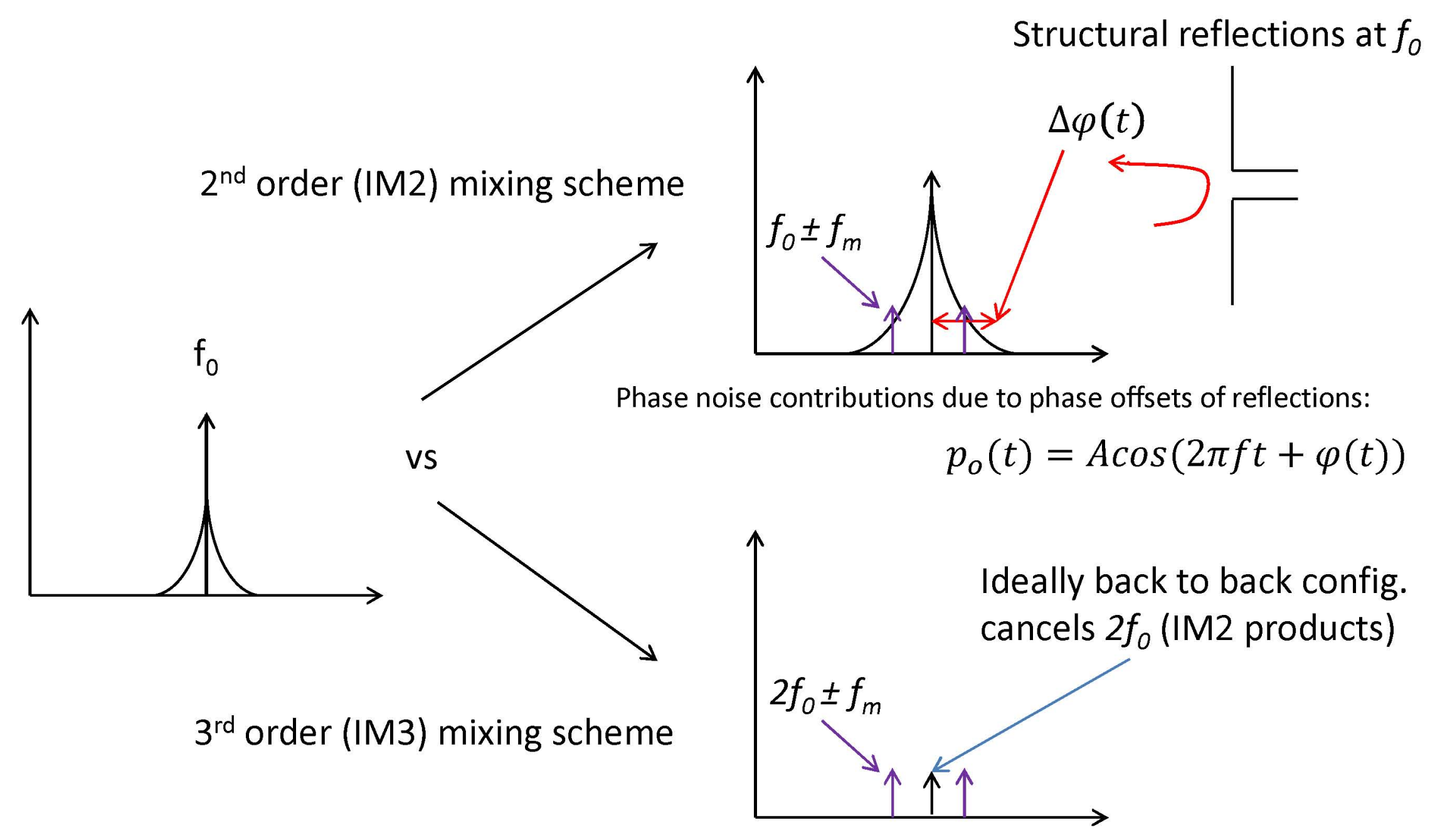




\section{Appendix III: Backscattering Link}

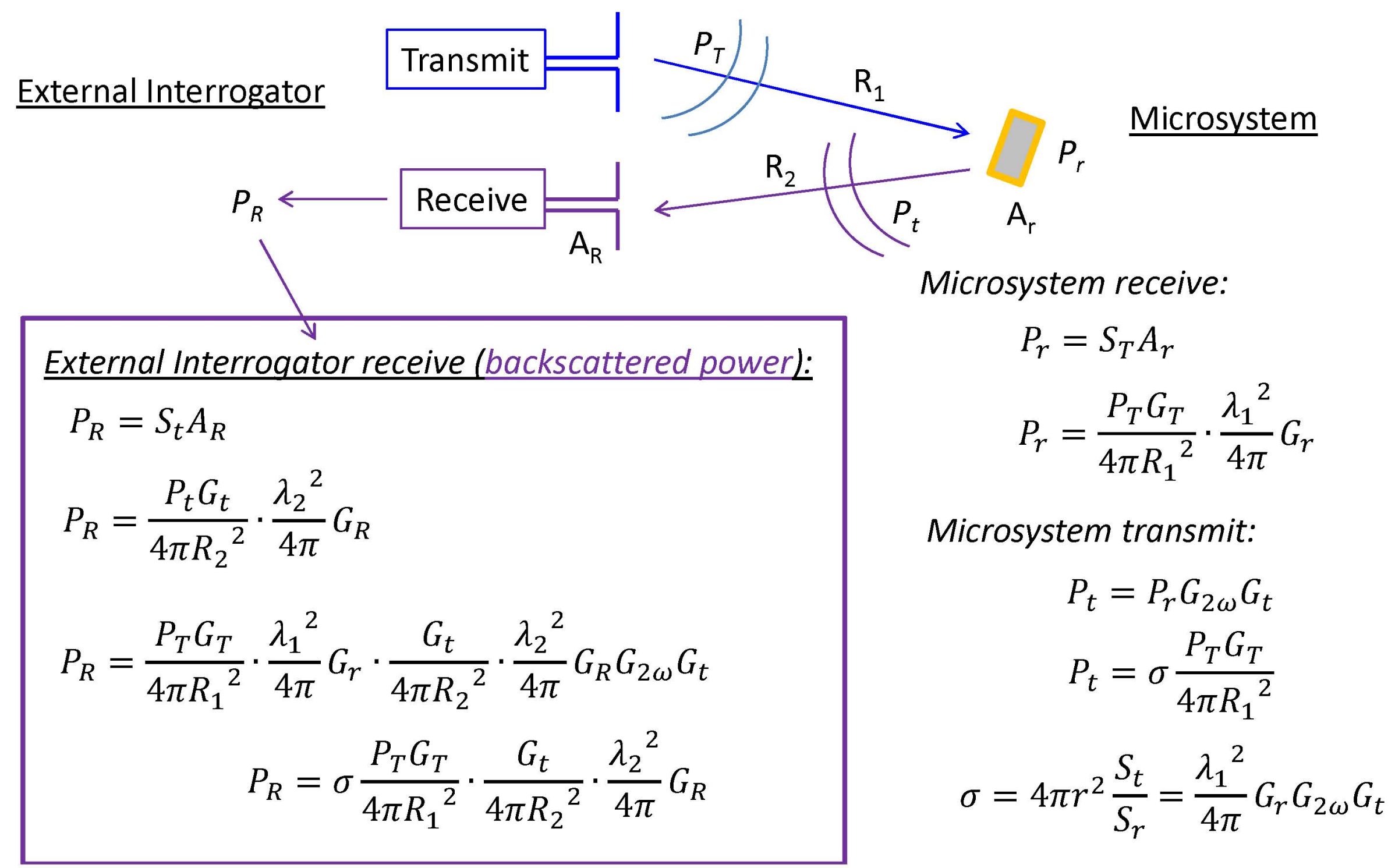




\section{Appendix IV: ADS Circuit Simulations}
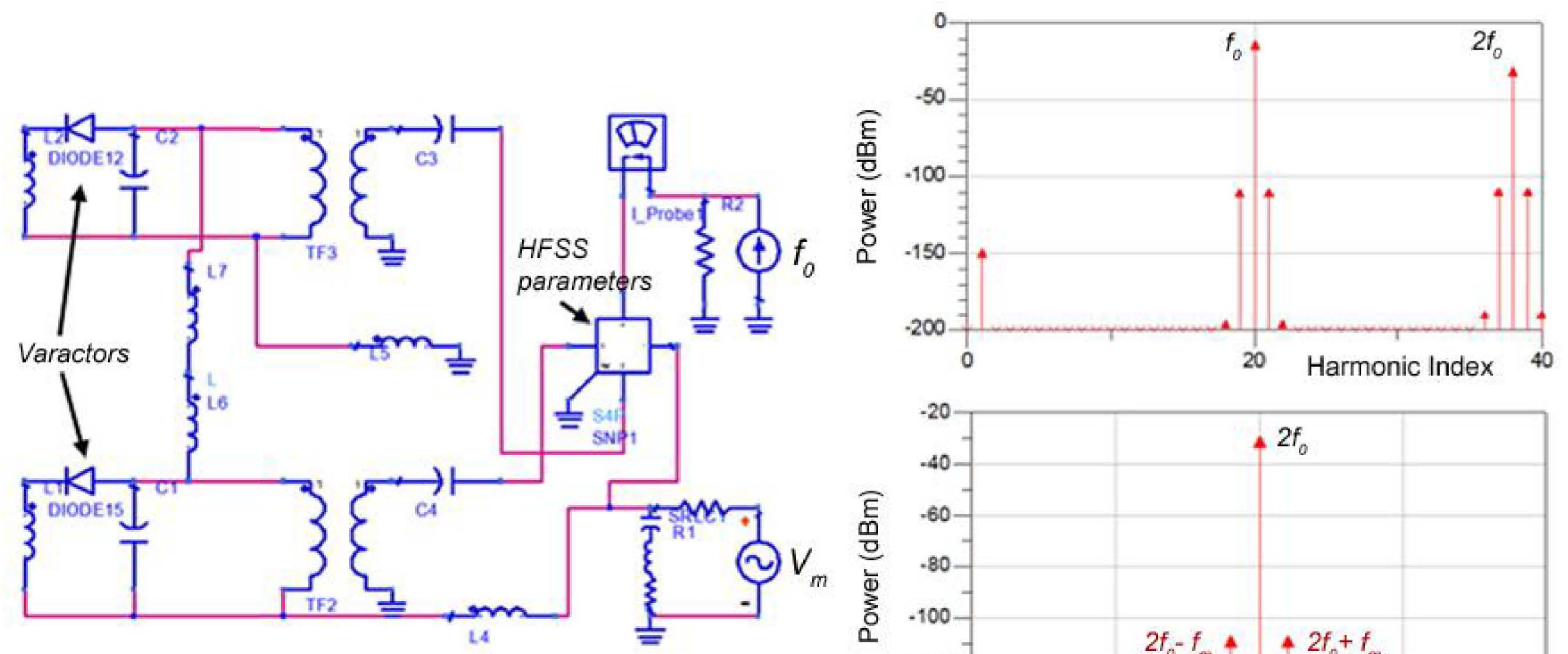

(a)

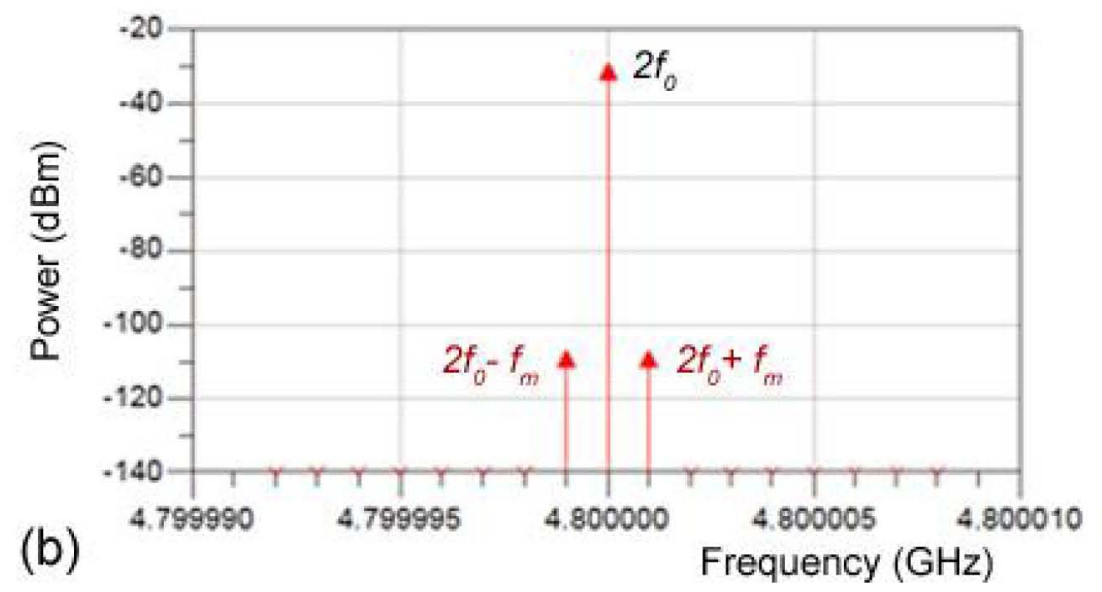

Simulated backscattering spectral response with $V_{m}$ of $100 \mu \mathrm{V}_{\mathrm{pp}}$ sinusoidal at $f_{m}$ (IF) of $1 \mathrm{kHz}$, incident radiated power, $P_{0}$, of $1 \mathrm{~mW}(0$ $\mathrm{dBm}$ ) at $f_{0}$ of $2.4 \mathrm{GHz}$. 


\section{Appendix IV(b): Detailed ADS}

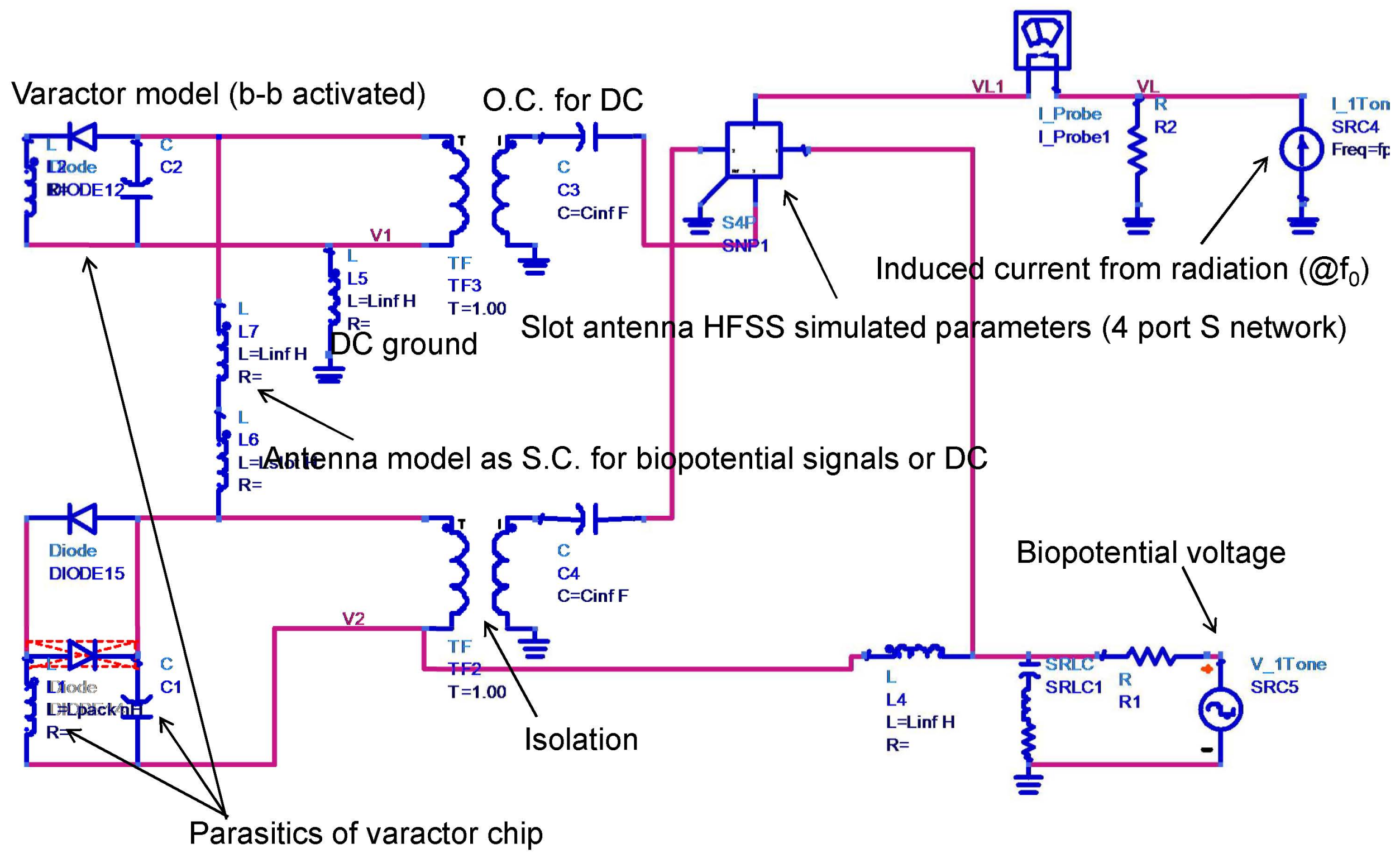




\section{Appendix V: Demodulator}

Simplified Block Diagram

Signal Generator

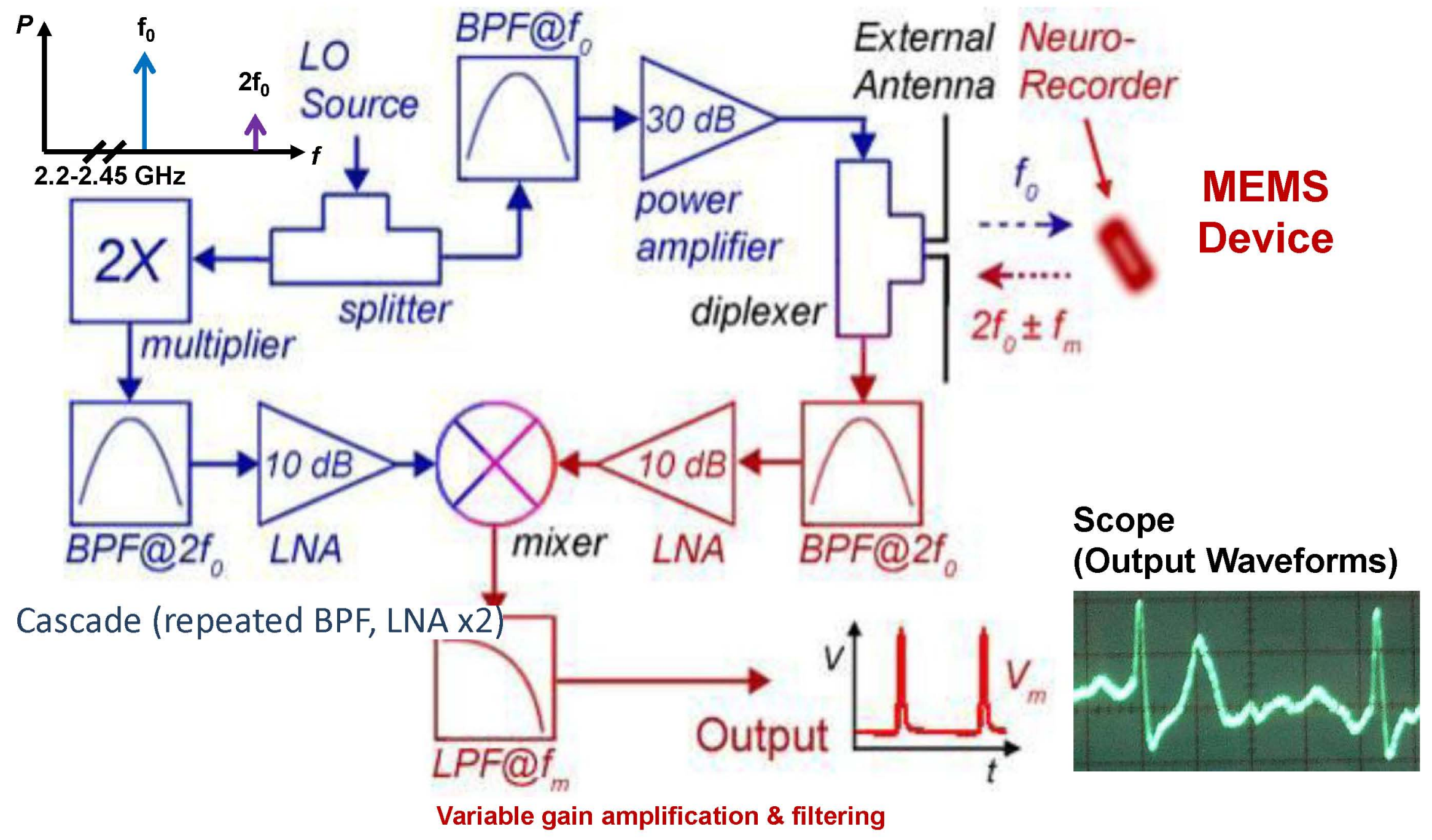

